\title{
In vitro teratogenicity testing using a 3D, embryo-like gastruloid system
}

\author{
Veronika Mantziou $^{\mathrm{a}, 1}$, Peter Baillie-Benson ${ }^{\mathrm{a}, 1,2}$, Manuela Jaklin $^{\mathrm{b}, \mathrm{c}, 1,4}$, Stefan Kustermann ${ }^{\mathrm{b}}$, \\ Alfonso Martínez Arias ${ }^{\mathrm{a}, 3}$, Naomi Moris ${ }^{\mathrm{a}, *, 2}$

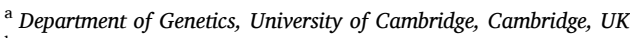 \\ ${ }^{\mathrm{b}}$ F. Hoffmann - La Roche, Pharma Research and Early Development, Roche Innovation Centre Basel, Switzerland \\ ${ }^{\mathrm{c}}$ Department for In Vitro Toxicology and Biomedicine Inaugurated by the Doerenkamp-Zbinden Foundation, University of Konstanz, Germany
}

\section{A R T I C L E I N F O}

Handling Editor: Dr. Bal-Price Anna

\section{Keywords:}

Gastruloids

Teratogenicity

Stem cells

Embryonic development

\begin{abstract}
A B S T R A C T
Pharmaceuticals intended for use in patients of childbearing potential need to be tested for teratogenicity before marketing. Several pharmaceutical companies use animal-free in vitro models which allow a more rapid selection of lead compounds and contribute to 3Rs principles ('replace, reduce and refine') by streamlining the selection of promising compounds submitted to further regulatory studies in animals. Currently available in vitro models typically rely on adherent monolayer cultures or disorganized 3D structures, both of which lack the spatiotemporal and morphological context of the developing embryo. A newly developed 3D 'gastruloid' model has the potential to achieve a more reliable prediction of teratogenicity by providing a robust recapitulation of gastrulation-like events alongside morphological coordination at relatively high-throughput. In this first proofof-concept study, we used both mouse and human gastruloids to examine a panel of seven reference compounds, with associated in vivo data and known teratogenic risk, to quantitatively assess in vitro teratogenicity. We observed several gross morphological effects, including significantly reduced elongation or decreased size of the gastruloids, upon exposure to several of the reference compounds. We also observed aberrant gene expression using fluorescent reporters, including SOX2, BRA, and SOX17, suggestive of multi-lineage differentiation defects and disrupted axial patterning. Finally, we saw that gastruloids recapitulated some of the known in vivo speciesspecific susceptibilities between their mouse and human counterparts. We therefore suggest that gastruloids represent a powerful tool for teratogenicity assessment by enabling relevant physiological recapitulation of early embryonic development, demonstrating their use as a novel in vitro teratogenic model system.
\end{abstract}

\section{Introduction}

Developing embryos are highly susceptible to any harmful exposure to teratogenic substances, due to incomplete epithelial barrier functions and detoxification capacity, especially in the first trimester of pregnancy $[1,2]$. Teratogens may cause congenital abnormalities to occur, leading to lifelong physical or functional impairment. However, the underlying mechanisms of many teratogens are still not well described and further investigations are necessary to enable us to understand, let alone predict, which disruptive effects might cause birth defects.

Particularly in the application of pharmaceuticals intended for women of childbearing potential, it is crucial to know which substances may bear teratogenic risks. Therefore, promising drug candidates must be screened for any developmental or fetal toxicity prior to clinical application. This involves testing in pregnant animals in order to predict the likely effect of compound exposure at different concentrations. However, a number of high-profile cases have suggested that model organisms like the mouse are often unable to accurately predict human teratogenicity, such as thalidomide [3,4]. As a result, Developmental and Reproductive Toxicology (DART) studies now incorporate testing on several species, including non-rodents such as rabbits or non-human primates, in order to have increased confidence in the safety of a new compound [5].

An alternative option, fast gaining traction in pharmaceutical

\footnotetext{
* Corresponding author.

E-mail address: naomi.moris@crick.ac.uk (N. Moris).

1 These authors contributed equally to the work.

2 Present address: The Francis Crick Institute, 1 Midland Rd, Somers Town, London, UK.

3 Present address: Systems Bioengineering, DCEXS, Universidad Pompeu Fabra, Doctor Aiguader, 88 ICREA, pág. Lluís Companys 23, Barcelona, Spain.

${ }^{4}$ Present address: Weleda AG, Dychweg 144144 Arlesheim, Switzerland
} 
Table 1

Administered compounds and their respective treatment concentrations.

\begin{tabular}{|c|c|c|c|}
\hline Compound & $\begin{array}{l}\text { Teratogenicity } \\
\text { classification }\end{array}$ & $\begin{array}{l}\text { Treatment } \\
\text { Concentrations [X1, } \\
\mathrm{X} 2, \mathrm{X} 3] \text { (mouse) }\end{array}$ & $\begin{array}{l}\text { Treatment } \\
\text { Concentrations [X1, } \\
\mathrm{X} 2,(\mathrm{X} 3)] \text { (human) }\end{array}$ \\
\hline $\begin{array}{l}\text { All-trans } \\
\text { retinoic } \\
\text { acid }\end{array}$ & Positive & $\begin{array}{l}0.4 \mathrm{nM}, 33 \mathrm{nM}, 100 \\
\mathrm{nM}\end{array}$ & $0.4 \mathrm{nM}, 33 \mathrm{nM}$ \\
\hline Valproic Acid & Positive & $\begin{array}{l}4 \mu \mathrm{M}, 333 \mu \mathrm{M}, 1000 \\
\mu \mathrm{M}\end{array}$ & $4 \mu \mathrm{M}, 333 \mu \mathrm{M}$ \\
\hline Bosentan & Positive & $4 \mu \mathrm{M}, 8 \mu \mathrm{M}, 18 \mu \mathrm{M}$ & $4 \mu \mathrm{M}, 18 \mu \mathrm{M}$ \\
\hline Thalidomide & Positive & $\begin{array}{l}0.4 \mu \mathrm{M}, 11 \mu \mathrm{M}, 100 \\
\mu \mathrm{M}\end{array}$ & $0.4 \mu \mathrm{M}, 100 \mu \mathrm{M}$ \\
\hline Phenytoin $^{\mathrm{a}}$ & Positive & $1 \mu \mathrm{M}, 5 \mu \mathrm{M}, 20 \mu \mathrm{M}$ & $20 \mu \mathrm{M}$ \\
\hline Ibuprofen & Positive & $\begin{array}{l}63 \mu \mathrm{M}, 120 \mu \mathrm{M}, 250 \\
\mu \mathrm{M}\end{array}$ & $63 \mu \mathrm{M}, 250 \mu \mathrm{M}$ \\
\hline Penicillin G & Negative & $63 \mu \mathrm{M}, 1 \mathrm{mM}, 2 \mathrm{mM}$ & $63 \mu \mathrm{M}, 1 \mathrm{mM}, 2 \mathrm{mM}$ \\
\hline
\end{tabular}

${ }^{\text {a }}$ Phenytoin was soluble in DMSO at $200 \mathrm{mM}$ but precipitates when mixed with $\mathrm{PBS}^{+/+}$, N2B27 or E6 medium at concentrations higher than $20 \mu \mathrm{M}$. Classification based on EMA ICH S5 (R3) guidelines on reproductive toxicology [5].

companies, is to utilize in vitro systems, such as pluripotent stem cells, to design assays for teratogenicity assessment [6]. The benefit of such systems is threefold: they facilitate human-specific predictions by using human cell lines; they reduce the number of animals required for research, thus contributing to a 3Rs principle (to 'replace, reduce and refine' animal use in research) and they allow for medium- to high-throughput analyses earlier in the drug development pipeline. As such, in vitro model systems that predict human teratogenicity represent a powerful new approach in pharmaceutical development.

Recently, several in vitro models have been developed and applied to the evaluation of developmental toxicants [7-10]. However, most of these models are established as adherent monolayer cultures, which are lacking an essential micro-physiological environment and the readouts of assays often only target a single germ layer. Therefore, commonly available in vitro systems do not fully reflect all the relevant features of spatial and temporal patterning or multi-lineage differentiation during gastrulation $[11,12]$. Instead, a more biologically relevant model that better recreates the native processes of early embryonic development, could reveal new mechanistic insights into disruptive events during embryogenesis and might improve prediction of teratogenicity.

Recent work has described a pluripotent stem cell (PSC)-based system that utilizes the self-organizing potential of PSCs to generate threedimensional structures that recapitulate elements of the early embryo
[13-15]. These are known as 'gastruloids', as they mirror some of the events of gastrulation; where the emergence of the three germ layers is coordinated along anterior-posterior, dorsal-ventral and medio-lateral axes to generate the elements of the body plan, from which the various tissues and organs will emerge. Studies have shown that these stem cell aggregates progressively break symmetry, undergo axial elongation and differentiate to all three germ layers (mesoderm, ectoderm and endoderm) in a manner that is spatially and temporally similar to the embryo. However, these structures do not contain anterior neural (brain) or extraembryonic cell types, meaning they do not have full organismal potential [16]. Recently, this work with mouse PSCs has been extended to the generation of gastruloids from human Embryonic Stem Cells (ESCs) [15]. These so-called, 'human gastruloids', recapitulate many features of the developing mammalian embryo, and may bring unique insights into human development with experimental tractability that overcomes several of the ethical and technical limitations of research on human embryos.

Gastruloids have reproducible morphological changes, germ layer proportions and spatiotemporal organization of gene expression that are easy to visualize and quantify. These are key benefits over traditional adherent cell methods or embryoid body (EB) cultures. They therefore offer an experimentally tractable system to explore the effect of a range of perturbations that may affect cell lineage differentiation, signaling, morphology, cell viability and growth $[13,15]$.

Here, we examine whether mouse and human gastruloids can have further application as an effective teratogenicity assay. Using a small reference panel of compounds; including all-trans retinoic acid, valproic acid, bosentan, thalidomide, phenytoin, ibuprofen and penicillin G; we explore gastruloid sensitivity to different concentrations and exposures. These are then compared between mouse and human gastruloids, against existing data from in vivo animal Embryo-Fetal Development (EFD) studies and compared to known human teratological status. We use a range of outputs including morphological shape descriptors, marker gene expression and cytotoxicity to qualitatively and quantitatively assess the effect of chemical exposure on gastruloids. Distribution of germ layer representative reporter gene expression of SOX2 (neuroectoderm), SOX17 (endoderm) and BRA (mesoderm) within human gastruloids, and $T / B r a$ in mouse gastruloids, enabled us to examine the correct generation of germ layer lineages and the extent of axial polarity. Furthermore, we compared the data with those from existing 3D mouse and human in vitro models to see whether the gastruloid systems are able to advance the physiological relevance compared to systems based on

\section{Mouse:}

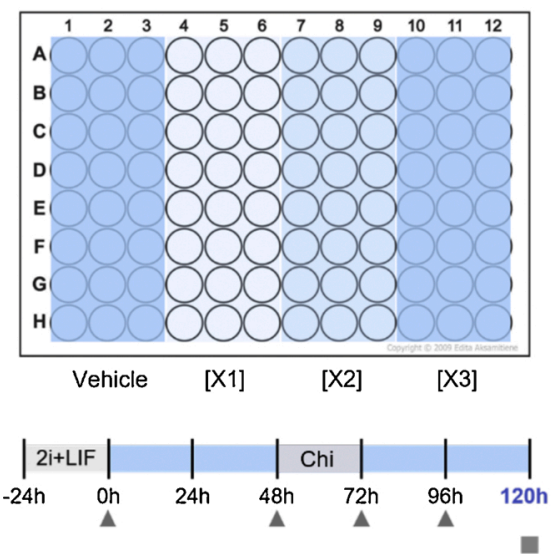

Human:

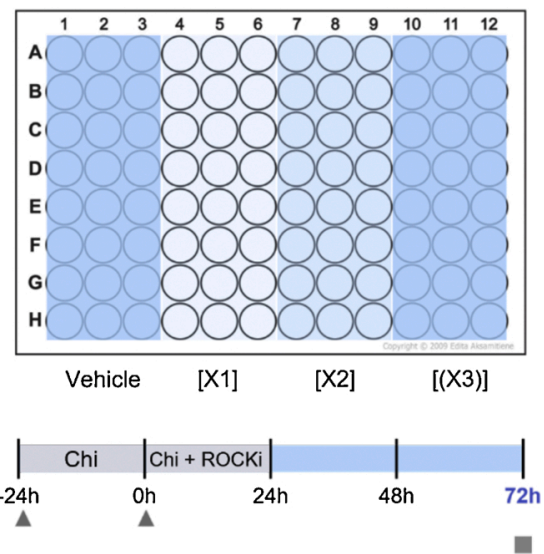

$\Delta$ Compounds administered

Imaging data collected

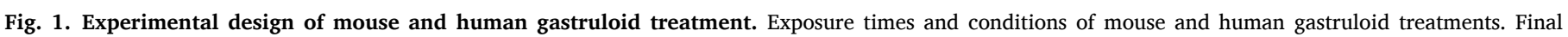

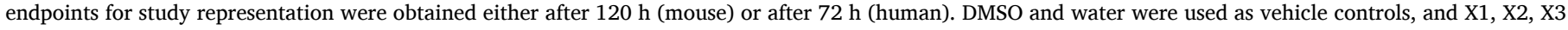
refer to the various treatment concentrations of the compounds (see Table 1). Chi, Chiron; ROCKi, ROCK inhibitor. 
Table 2

Summary of observed effects. (For interpretation of the references to colour in this table, the reader is referred to the web version of this article).

\begin{tabular}{|c|c|c|c|c|c|c|}
\hline & \multicolumn{3}{|c|}{ Mouse Gastruloids } & \multicolumn{3}{|c|}{ Human Gastruloids } \\
\hline & $0.4 \mathrm{nM}$ & $33 \mathrm{nM}$ & $100 \mathrm{nM}$ & $0.4 \mathrm{nM}$ & \multirow{2}{*}{\multicolumn{2}{|c|}{$\begin{array}{l}\text { Morphological effect; Size } \\
\text { reduction, Gene expression } \\
\text { effect }\end{array}$}} \\
\hline $\begin{array}{c}\text { Retinoic } \\
\text { Acid }\end{array}$ & $\begin{array}{l}\text { Morphologic } \\
\text { al effect, } \\
\text { Gene } \\
\text { expression } \\
\text { effect }\end{array}$ & $\begin{array}{l}\text { Morphologic } \\
\text { al effect; Size } \\
\text { reduction; } \\
\text { Suspected } \\
\text { cytotoxicity, } \\
\text { Gene } \\
\text { expression } \\
\text { effect }\end{array}$ & $\begin{array}{l}\text { Morphologic } \\
\text { al effect; Size } \\
\text { reduction; } \\
\text { Suspected } \\
\text { cytotoxicity, } \\
\text { Gene } \\
\text { expression } \\
\text { effect }\end{array}$ & $\begin{array}{c}\text { Small } \\
\text { morphologica } \\
\text { l effect }\end{array}$ & & \\
\hline & $4 \mu \mathrm{M}$ & $333 \mu \mathrm{M}$ & $1 \mathbf{m M}$ & $4 \mu \mathrm{M}$ & \multicolumn{2}{|c|}{$333 \mu \mathrm{M}$} \\
\hline $\begin{array}{c}\text { Valproic } \\
\text { Acid }\end{array}$ & $\begin{array}{c}\text { Small } \\
\text { morphologica } \\
\text { l effect }\end{array}$ & $\begin{array}{l}\text { Morphologic } \\
\text { al effect; Size } \\
\text { reduction; } \\
\text { Suspected } \\
\text { cytotoxicity, } \\
\text { Gene } \\
\text { expression } \\
\text { effect }\end{array}$ & $\begin{array}{l}\text { Morphologic } \\
\text { al effect; Size } \\
\text { reduction; } \\
\text { Suspected } \\
\text { cytotoxicity, } \\
\text { Gene } \\
\text { expression } \\
\text { effect }\end{array}$ & $\begin{array}{c}\text { Minimal } \\
\text { effect }\end{array}$ & \multicolumn{2}{|c|}{$\begin{array}{l}\text { Morphological effect; Size } \\
\text { reduction, Gene expression } \\
\text { effect }\end{array}$} \\
\hline & $4 \mu \mathrm{M}$ & $8 \mu \mathrm{M}$ & $18 \mu \mathrm{M}$ & $4 \mu \mathrm{M}$ & \multicolumn{2}{|c|}{$18 \mu \mathrm{M}$} \\
\hline Bosentan & $\begin{array}{c}\text { Minimal } \\
\text { effect }\end{array}$ & $\begin{array}{l}\text { Minimal } \\
\text { effect }\end{array}$ & $\begin{array}{c}\text { Minimal } \\
\text { effect }\end{array}$ & $\begin{array}{l}\text { Minimal } \\
\text { effect }\end{array}$ & \multicolumn{2}{|c|}{$\begin{array}{l}\text { Morphological effect; Size } \\
\text { reduction, Gene expression } \\
\text { effect }\end{array}$} \\
\hline & $0.4 \mu \mathrm{M}$ & $11 \mu \mathrm{M}$ & $100 \mu \mathrm{M}$ & $0.4 \mu \mathrm{M}$ & \multicolumn{2}{|c|}{$100 \mu \mathrm{M}$} \\
\hline $\begin{array}{l}\text { Thali- } \\
\text { domide }\end{array}$ & No effect & No effect & $\begin{array}{l}\text { Gene } \\
\text { expression } \\
\text { effect }\end{array}$ & $\begin{array}{l}\text { Morphologic } \\
\text { al effect; Size } \\
\text { reduction, } \\
\text { Gene } \\
\text { expression } \\
\text { effect }\end{array}$ & \multicolumn{2}{|c|}{$\begin{array}{l}\text { Morphological effect; Size } \\
\text { reduction, Gene expression } \\
\text { effect }\end{array}$} \\
\hline & $\mathbf{1} \mu \mathrm{M}$ & $5 \mu \mathrm{M}$ & $20 \mu \mathrm{M}$ & $20 \mu \mathrm{M}$ & \multicolumn{2}{|c|}{ - } \\
\hline Phenytoin & No effect & $\begin{array}{l}\text { Gene } \\
\text { expression } \\
\text { effect }\end{array}$ & $\begin{array}{l}\text { Gene } \\
\text { expression } \\
\text { effect }\end{array}$ & $\begin{array}{l}\text { Minimal } \\
\text { effect }\end{array}$ & \\
\hline & $63 \mu \mathrm{M}$ & $120 \mu \mathrm{M}$ & $250 \mu \mathrm{M}$ & $63 \mu \mathrm{M}$ & \multicolumn{2}{|c|}{$250 \mu \mathrm{M}$} \\
\hline Ibuprofen & $\begin{array}{l}\text { Gene } \\
\text { expression } \\
\text { effect }\end{array}$ & $\begin{array}{l}\text { Gene } \\
\text { expression } \\
\text { effect }\end{array}$ & $\begin{array}{l}\text { Gene } \\
\text { expression } \\
\text { effect }\end{array}$ & $\begin{array}{l}\text { Morphologic } \\
\text { al effect; Size } \\
\text { reduction, } \\
\text { Gene } \\
\text { expression } \\
\text { effect }\end{array}$ & \multicolumn{2}{|c|}{$\begin{array}{l}\text { Morphological effect; Size } \\
\text { reduction, Gene expression } \\
\text { effect }\end{array}$} \\
\hline & $63 \mu \mathrm{M}$ & $1 \mathrm{mM}$ & $2 \mathrm{mM}$ & $63 \mu \mathrm{M}$ & $1 \mathrm{mM}$ & $2 \mathrm{mM}$ \\
\hline Penicillin G & $\begin{array}{c}\text { Minimal } \\
\text { effect }\end{array}$ & $\begin{array}{c}\text { Small } \\
\text { morphologica } \\
\text { l effect }\end{array}$ & $\begin{array}{c}\text { Small } \\
\text { morphologica } \\
\text { l effect }\end{array}$ & $\begin{array}{c}\text { Minimal } \\
\text { effect }\end{array}$ & $\begin{array}{c}\text { Minimal } \\
\text { effect }\end{array}$ & No effect \\
\hline
\end{tabular}

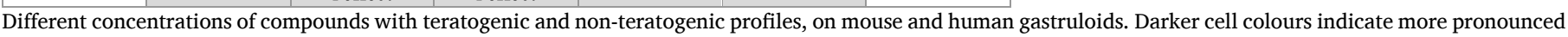
effects.

morphologically less structured embryoid bodies $[7,17,18]$.

\section{Materials and methods}

\subsection{Mouse embryonic stem cell culture}

Mouse embryonic stem cells were maintained as previously described, on gelatin-precoated tissue culture plastic in ES + LIF (leukemia inhibitory factor) medium [19]. Cells were passaged at a density of $8 \times 10^{3}$ cells $/ \mathrm{cm}^{2}$ every second day, with approximately two-thirds of the culture medium exchanged for fresh ES + LIF medium on the intervening days. The cell lines used in this study were E14Tg2A [20] and T/Bra::GFP [21]. They were maintained in culture for at least two passages post-thawing prior to experimental use and they were propagated for no more than 30 passages in vitro. All cell counting during routine maintenance and experimentation was performed with an ORFLO Moxi Z mini automated cell counter (ORFLO Technologies, MXZ002).

\subsection{Mouse gastruloid culture}

Gastruloids were prepared by aggregating 300 mouse embryonic stem cells in $40 \mu \mathrm{l}$ droplets of N2B27 (NDiff ${ }^{\circledR}$ 227, Takara Bio Inc. Y40002) per well of U-bottomed 96-well plates (Greiner 650185). The full protocol for generating mouse gastruloids can be found on Protocol Exchange (DOI:https://doi.org/10.1038/protex.2018.094) and a full characterisation has been described previously [13]. The following modifications were made to the protocol. Cell cultures were plated into new flasks at a density of $8 \times 10^{3}$ cells $/ \mathrm{cm}^{2}$ in ES + LIF medium two days prior to preparing gastruloids. The culture medium was changed fully to $2 \mathrm{i}+\mathrm{LIF}$ medium the following day as a $24 \mathrm{~h}$ pre-treatment.

The compounds were administered to the gastruloids on plating and daily after aggregation was complete (at $0 \mathrm{~h}, 48 \mathrm{~h}, 72 \mathrm{~h}$ and $96 \mathrm{~h}$ postplating). Between 48 and $72 \mathrm{~h}$, gastruloids were exposed to CHIR99,021 , as described previously [13]. The compounds were prepared fresh from frozen stocks in N2B27 culture medium at each time point. 

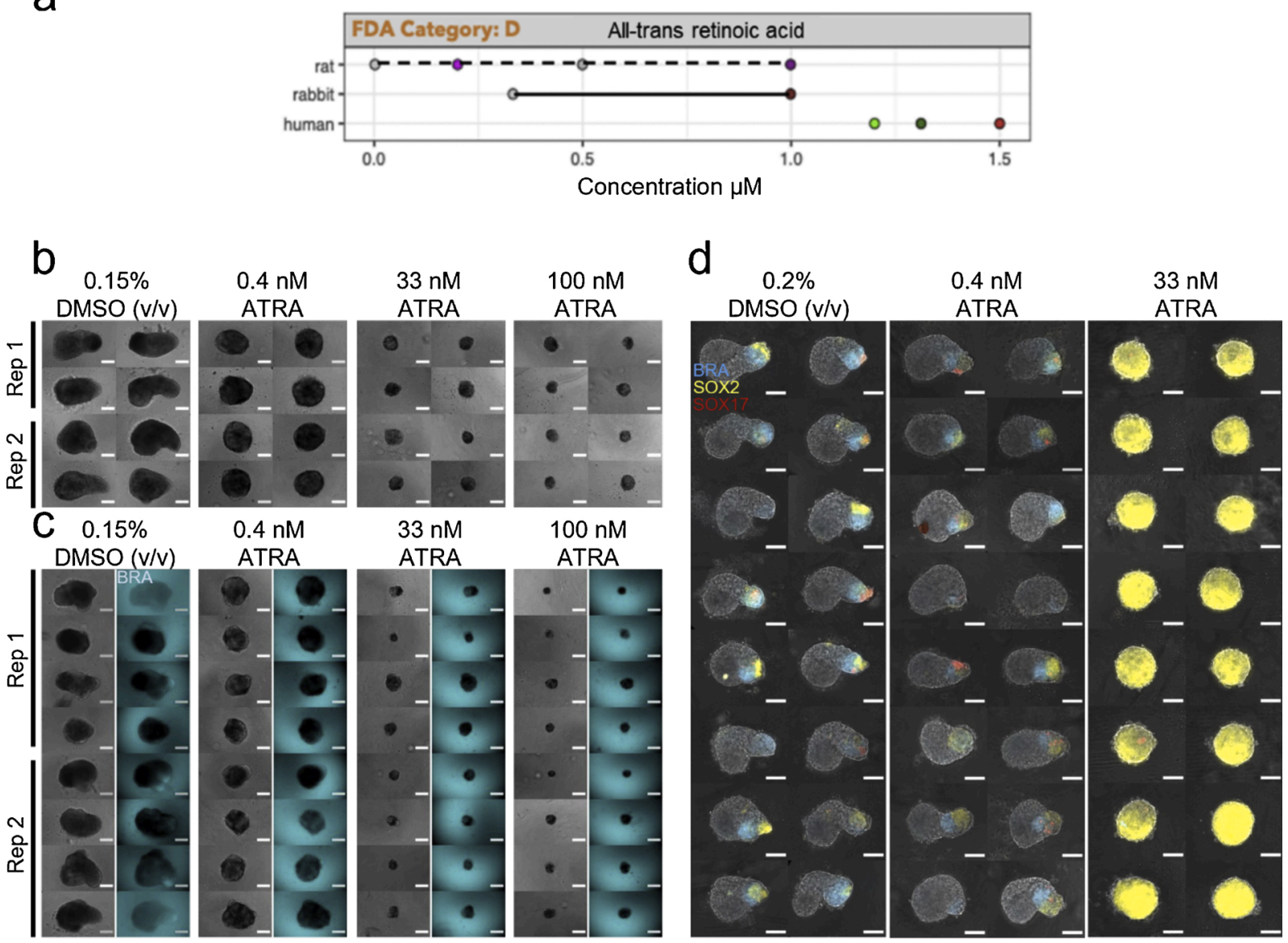

Fig. 2. Gastruloids following all-trans retinoic acid exposure. (a) Literature-based exposure limits in different species in $[\mu \mathrm{M}]$ (o/o no effect/ NOAEL; o/o teratogenic/ LOAEL, see Suppl. Fig. S1). (b-c) E14Tg2A (b) and T/Bra::GFP (c) mouse gastruloids at $120 \mathrm{~h}$, following exposure to DMSO (vehicle control) or all-trans retinoic acid (ATRA). Color indicates fluorescent expression of T/Bra::GFP (blue). Representative images from 2 replicates shown. (d) RUES2-GLR human gastruloids at $72 \mathrm{~h}$, following exposure to DMSO or ATRA. Color indicates fluorescent expression of BRA-mCerulean (blue), SOX2-mCitrine (yellow) and SOX17-tdTomato (red). Representative images from 1 replicate shown. Scale bars represent $200 \mu \mathrm{m}$ (b,c) and $100 \mu \mathrm{m}$ (d). Rep, Replicate. (For interpretation of the references to colour in this figure legend, the reader is referred to the web version of this article).

\subsection{Human embryonic stem cell culture}

The human cell line used in this study was the ES cell line RUES2GLR (mCit-SOX2, mCerulean-BRA, tdTomato-SOX17) [22]. All cells were cultured in humidified incubators at $37{ }^{\circ} \mathrm{C}$ and $5 \% \mathrm{CO}_{2}$. Human ES cells were cultured routinely in Nutristem hPSC XF medium (Biological Industries, 05-100-1A) on $0.5 \mu \mathrm{g} / \mathrm{cm}^{2}$ Vitronectin-coated plates (Gibco, A14700). Cells were passaged using $0.5 \mathrm{mM}$ EDTA in phosphate-buffered saline without $\mathrm{Mg}^{2+}$ or $\mathrm{Ca}^{2+}\left(\mathrm{PBS}^{-/-}\right.$) (Invitrogen, 15575-038).

\subsection{Human gastruloid culture}

The full protocol for generating human gastruloids can be found on Protocol Exchange (DOI: 10.21203/rs.3.pex-812/v1) and a full characterisation of the structures has been previously described [15]. Before human gastruloid culture, cells were passaged to single cell suspension and plated to achieve 65,000 cells per well of a 6-well plate in Nutristem supplemented with 1:2,000 Y-27632 (ROCK inhibitor; Sigma Aldrich, Y0503) [23]. Adherent cultures were pre-treated in Nutristem supplemented with $3.25 \mu \mathrm{M}$ CHIR99021 (Chiron; Tocris Biosciences, 4423) and either $0.2 \%$ DMSO (vehicle), water (vehicle for penicillin G) or the different compound concentrations on the fourth day of adherent culture. After pre-treatment for $24 \mathrm{~h}$, cells were dissociated using 0.5 mM EDTA in PBS $^{-/-}$(Invitrogen, 15575-038), washed in $\mathrm{PBS}^{-/-}$and reaggregated in basal differentiation medium, Essential 6 (E6; Thermo Fisher Scientific, A15165-01), supplemented with 1:2,000 Y-27632 (ROCK inhibitor), $0.5 \mu \mathrm{M}$ Chiron and either $0.2 \% \mathrm{DMSO}$, water (vehicles) or the different compound concentrations. Cell numbers were determined using an automated cell counter (Moxi Z Mini, ORFLO Technologies, MXZ002) and 400 cells per $40 \mu 1$ were added to each well of an ultra-low-adherence 96-well plate (CellStar, 650970). The cell suspension was centrifuged using a benchtop plate centrifuge (Eppendorf) at $700 \mathrm{rpm}$ at room temperature for $2 \mathrm{~min}$. The following day, 150 $\mu \mathrm{l}$ fresh E6 medium was added to each well. The medium was exchanged for fresh E6 medium daily after this time point (150 $\mu$ l per well).

\subsection{Embryoid body based in vitro systems}

To evaluate the data from the gastruloid models, described protocols of the mouse embryonic stem cell test (mEST) [17] and a human 3D in vitro model [18] were used with comparable compound concentrations. Both of the in vitro systems depend on the application of three-dimensional embryoid body cultures derived from either mouse embryonic stem cells (mESC, ES-D3, ATCC, CRL-1934) or human episomal induced pluripotent stem cells (hiPSC, Gibco, A18945). The 
a
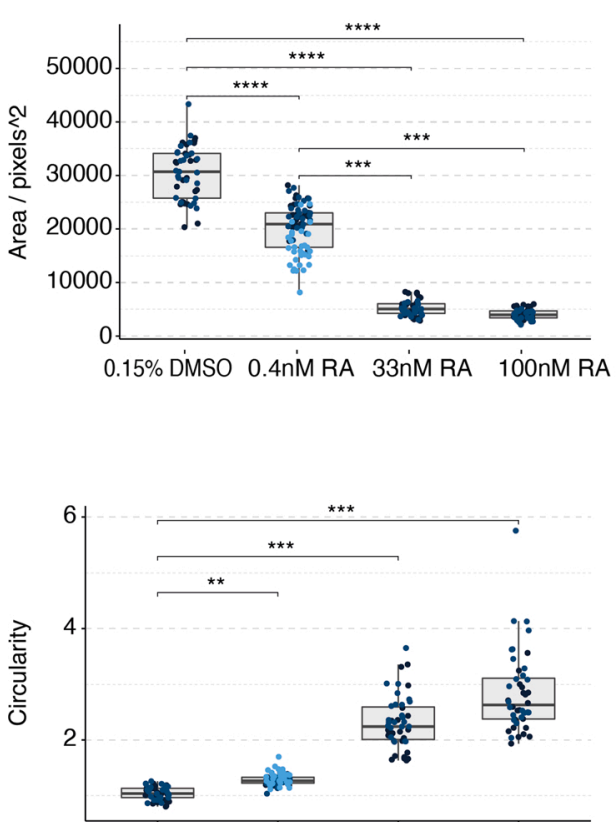

0.15\% DMSO 0.4nM RA 33nM RA 100nM RA

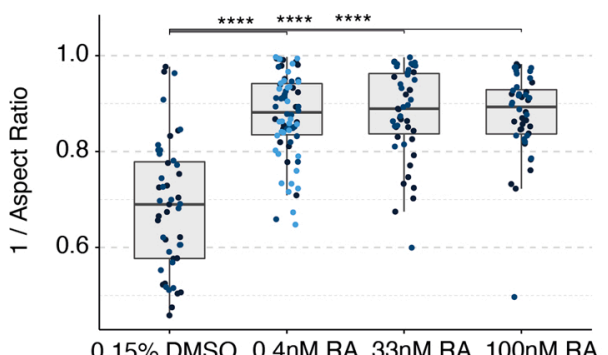

b

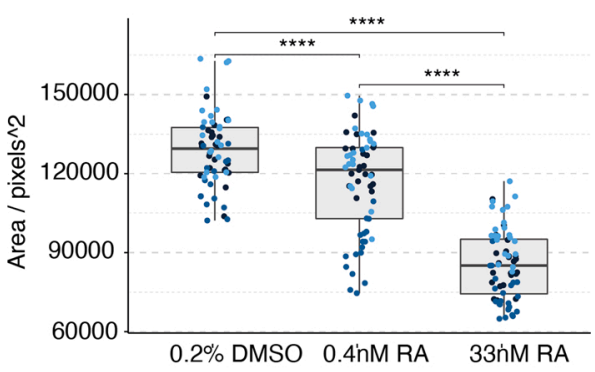

Fig. 3. Gastruloid quantification following all-trans retinoic acid exposure. Quantification of morphology of mouse gastruloids (a), and human gastruloids (b) including Area (top), Circularity (middle) and 1/Aspect Ratio (bottom). Dot colors indicate experimental replicates, and boxplots indicate spread of the data. Significant differences between DMSO (vehicle control) and all-trans retinoic acid (ATRA) treatment conditions are indicated in the plots (One-way ANOVA, Tukey's post-hoc test, a = 0.05 indicated by asterisks (see Materials and Methods for thresholds)).

Table 3

Effective concentrations.

\begin{tabular}{|c|c|c|c|c|c|c|}
\hline \multirow[b]{2}{*}{ Compound } & \multicolumn{3}{|l|}{ Mouse } & \multicolumn{3}{|l|}{ Human } \\
\hline & mEST ID $_{50}$ & mouse Gastruloid LOAEL & rat in vivo LOAEL Cmax & hiPSC- derived EBs $\mathrm{TC}_{20}$ & Human Gastruloid LOAEL & human in vivo Cmax \\
\hline Retinoic Acid & $3.7 \mathrm{nM}$ & $0.4 \mathrm{nM}$ & $1 \mu \mathrm{M}$ & $<0.1 \mathrm{nM}$ & $0.4 \mathrm{nM}$ & $1.31 \mu \mathrm{M}$ \\
\hline Valproic Acid & $589 \mu \mathrm{M}$ & $333 \mu \mathrm{M}$ & $1.6 \mathrm{mM}$ & $85 \mu \mathrm{M}$ & $333 \mu \mathrm{M}$ & $1.4 \mathrm{mM}$ \\
\hline Bosentan & $68.2 \mu \mathrm{M}$ & $\mathrm{n} / \mathrm{a}$ & $30 \mu \mathrm{M}$ & $13 \mu \mathrm{M}$ & $18 \mu \mathrm{M}$ & $\mathrm{n} / \mathrm{a}$ \\
\hline Thalidomide & $\mathrm{n} / \mathrm{a}$ & $100 \mu \mathrm{M}$ & $19 \mu \mathrm{M}$ & $<0.1 \mu \mathrm{M}$ & $0.4 \mu \mathrm{M}$ & $2.4 \mu \mathrm{M}$ \\
\hline Phenytoin & $0.39 \mu \mathrm{M}$ & $5 \mu \mathrm{M}$ & $106 \mu \mathrm{M}$ & $<8 \mu \mathrm{M}$ & $\mathrm{n} / \mathrm{a}$ & $57 \mu \mathrm{M}$ \\
\hline Ibuprofen & $49 \mu \mathrm{M}$ & $63 \mu \mathrm{M}$ & $1.6 \mathrm{mM}$ & $855 \mu \mathrm{M}$ & $63 \mu \mathrm{M}$ & $286 \mu \mathrm{M}$ \\
\hline Penicillin G & $\mathrm{n} / \mathrm{a}$ & $\mathrm{n} / \mathrm{a}$ & $\mathrm{n} / \mathrm{a}$ & $\mathrm{n} / \mathrm{a}$ & $\mathrm{n} / \mathrm{a}$ & $\mathrm{n} / \mathrm{a}$ \\
\hline
\end{tabular}

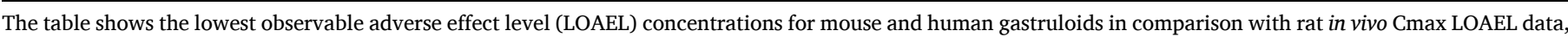

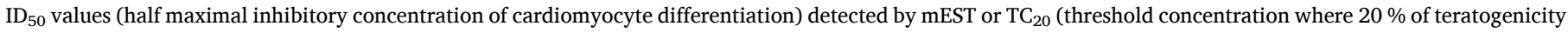
was detected by differential expression) from hiPSC-derived EBs, and the maximum human therapeutic plasma concentrations (Cmax) [5].

teratogenicity determination of the MEST is based on the half maximal inhibition of differentiation into beating cardiomyocytes $\left(\mathrm{ID}_{50}\right)$ and the human model focuses on the assessment of differential gene expression of early developmental markers within non-cytotoxic concentration ranges $\left(\mathrm{TC}_{20}\right)$.

\subsection{Compound application and experimental design}

All compounds were reconstituted in sterile-filtered DMSO (SigmaAldrich D2438) except for penicillin, which was diluted in water and then stored at $-20{ }^{\circ} \mathrm{C}$ (see Table 1 ). The vehicle treatments used a concentration of DMSO or water equivalent to that of the highest DMSO or 

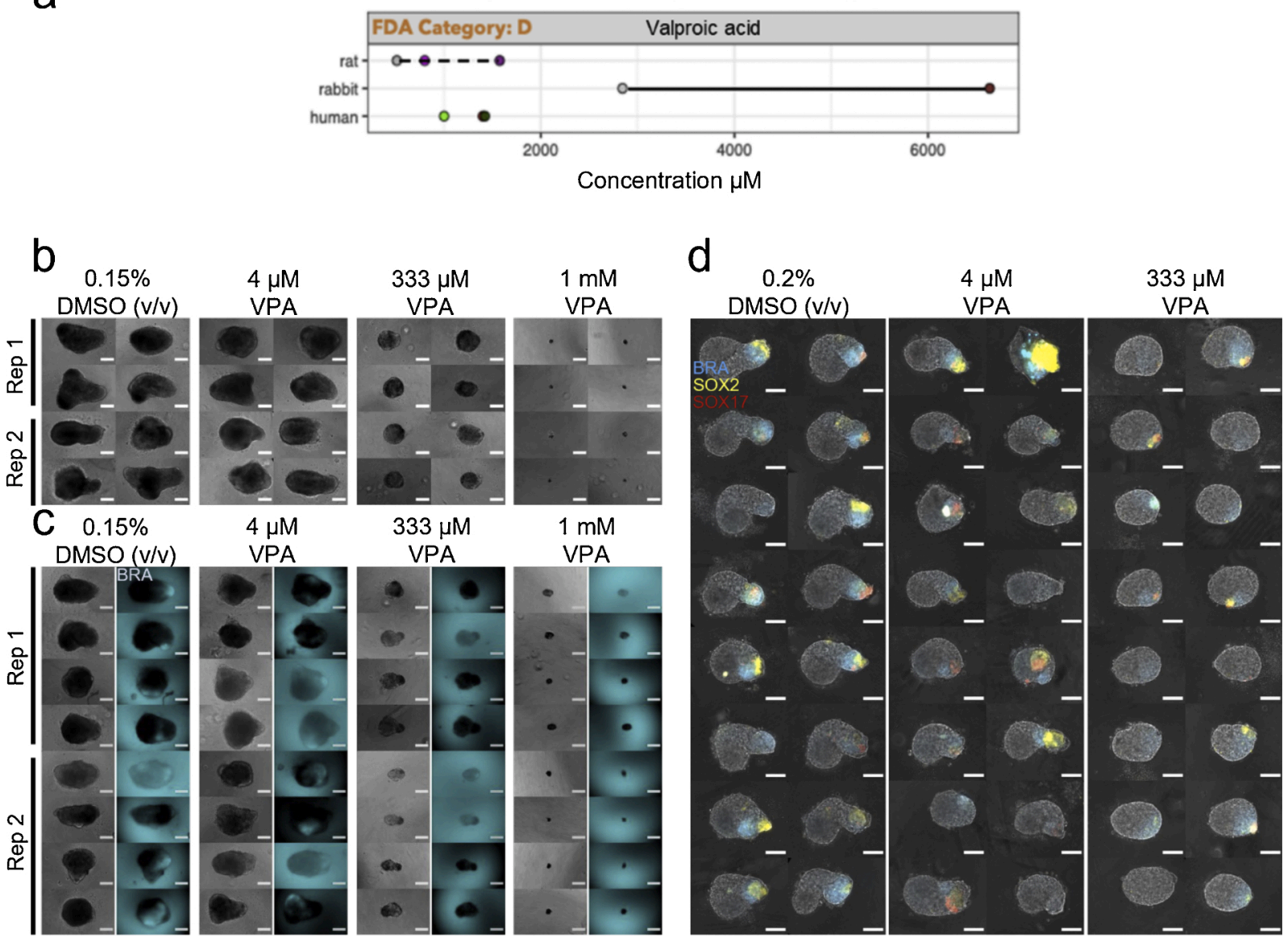

Fig. 4. Gastruloids following valproic acid exposure. (a) Literature-based exposure limits in different species in [ $\mu \mathrm{M}]$ (o/o no effect/ NOAEL; o/o teratogenic/ LOAEL, see Suppl. Fig. S1). (b-c) E14Tg2A (b) and T/Bra::GFP (c) mouse gastruloids at $120 \mathrm{~h}$, following exposure to DMSO (vehicle control) or valproic acid (VPA). Color indicates fluorescent expression of T/Bra::GFP (blue). Representative images from 2 replicates shown. (d) RUES2-GLR human gastruloids at 72 h, following exposure to DMSO or valproic acid. Color indicates fluorescent expression of BRA-mCerulean (blue), SOX2-mCitrine (yellow) and SOX17-tdTomato (red). Representative images from 1 replicate shown. Scale bars represent $200 \mu \mathrm{m}$ (b,c) and $100 \mu \mathrm{m}$ (d). Rep, Replicate. (For interpretation of the references to colour in this figure legend, the reader is referred to the web version of this article).

water concentration of the corresponding compound treatments. This ranged from $0.1-0.2 \%$ by volume DMSO and never exceeded $0.25 \%$, and $0.8 \%$ by volume water. We used a small panel of compounds (Table 1) which represent both positive (all-trans retinoic acid, valproic acid, bosentan, thalidomide, phenytoin, ibuprofen) and negative (penicillin G) references. Classifications are based on data from ICH S5 (R3) guidelines [5] (Suppl. Fig. S1). Each experiment was repeated 3 times for the RUES2-GLR and E14Tg2A cell lines and at least twice for the T/Bra::GFP line. The 96-well plate design, compound administration and timing of exposure can be seen in Fig. 1. Widefield imaging data, describing both morphological shape changes over time and fluorescent reporter expression, was collected at $120 \mathrm{~h}$ time points in the mouse system, and at $72 \mathrm{~h}$ time points in the human system. The reason for these timepoints is that they represent the end-point of each gastruloid culture, at which the maximal cell type diversity and axial elongation is observed, and are therefore likely to be functionally equivalent.

\subsection{Microscopy and image analysis}

Live imaging was conducted with a Nikon Ti-E inverted widefield microscope equipped with a cooled CMOS camera (Orca Flash 4.0, Hamamatsu) with appropriate environmental controls $\left(37{ }^{\circ} \mathrm{C}, 5 \% \mathrm{CO}_{2}\right.$; Okolab) and a Nikon pE-100 white LED light source for phase contrast imaging. The T/Bra::GFP and RUES2-GLR fluorescent reporter lines were visualised with an LED light source (Lumencor ${ }^{\circledR}$ Spectra X) and appropriate filters (Cerulean: Blue - 434/17, SPX-CFP-2432C-NTE FF01EM483/32 FF458-Di-02; GFP: Cyan - 475/28 SPX-GFP-1828A-NTE FF02-EM520/28 FF495-Di-03; YFP: Teal - 510/10 SPX-YFP-2427BNTE FF01-EM542/27 FF520-Di-02; tdTomato: Red - 632/22 SPXTxRed-4040C-NTE FF01-EM624/40 FF593-Di03). A 10x objective (CFI Achromat ADL, N.A. 0.25, W.D. $6.2 \mathrm{~mm}$, Ph1; cat. no. MRP40102) was used for the E14Tg2A and T/Bra::GFP mouse ES cell lines, while a 20x objective (CFI SuperPlanFluor ELWD ADM, N.A. 0.45, W.D. 8.2-6.9 $\mathrm{mm}$, correction collar at $1.2 \mathrm{~mm}$, Ph1; cat. no. MRH48230) was used for the RUES2-GLR human ES cell line. Images were acquired through the Nikon NIS-Elements AR software platform (4.30.02 (Build 1053) LO 64bit, running on Windows 7 with associated drivers controlling the light source and for multidimensional imaging). Imaging datasets were first converted to TIFF format with NIS-Elements, before further manipulation with the FIJI ImageJ distribution to collate montages per condition. Images of the mouse gastruloids were then cropped and the bit-depth of the images of both human and mouse gastruloids was reduced with custom ImageJ macros (details available at https://github.com/naomimoris/humanGastruloids_shapeDescriptors). The 8-bit TIFF files were passed through a custom analysis pipeline, implemented in the Project Jupyter Python 3.6 distribution [23]. Input files of the mouse gastruloids 
a

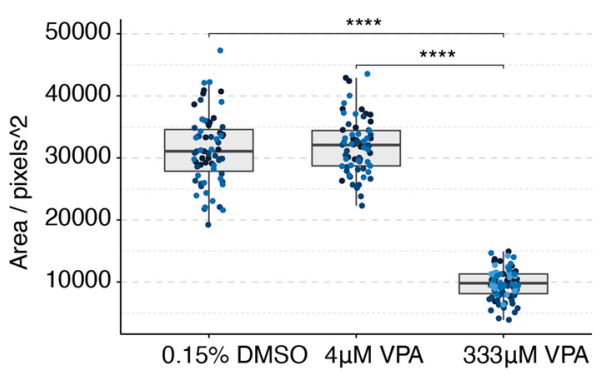

b

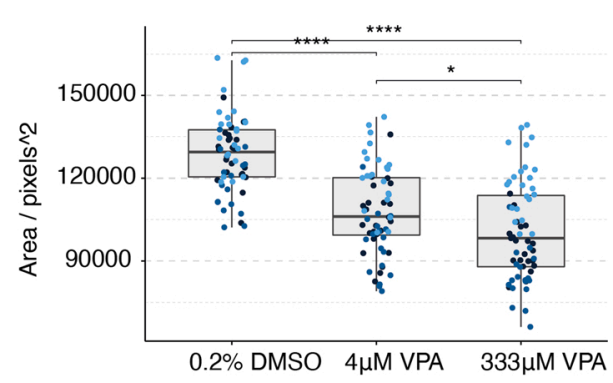

Fig. 5. Gastruloid quantification following valproic acid exposure. Quantification of morphology of mouse gastruloids (a), and human gastruloids (b) including Area (top), Circularity (middle) and 1/Aspect Ratio (bottom). Dot colors indicate experimental replicates, and boxplots indicate spread of the data. Significant differences between DMSO (vehicle control) and valproic acid (VPA) treatment conditions are indicated in the plots (One-way ANOVA, Tukey's post-hoc test, $a=0.05$ indicated by asterisks (see Materials and Methods for thresholds)).
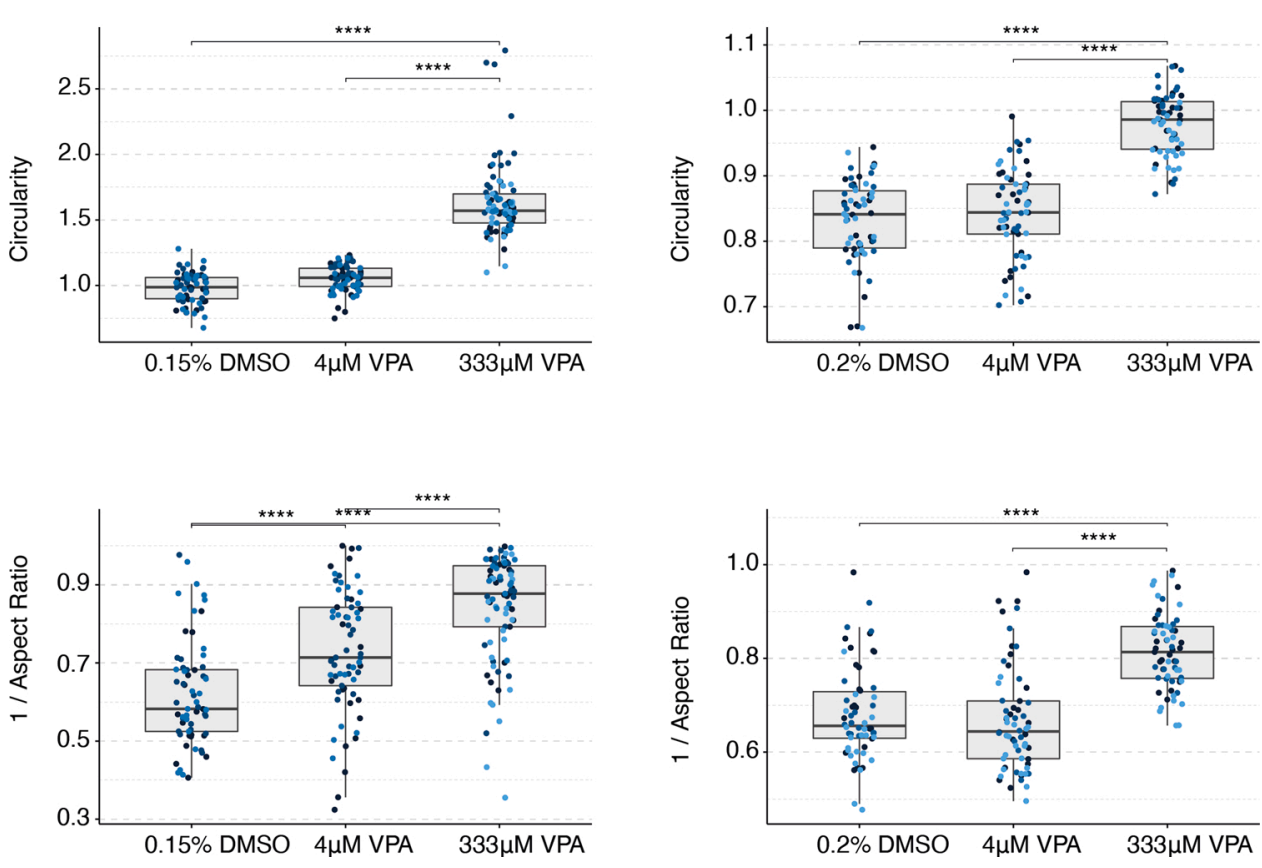

were cropped to a size that favored optimal performance of the computer vision methods used by the pipeline. Quantification contours were checked manually and the resulting measurements were curated by removing any measurements where the contours had failed to describe the shape of the human and mouse gastruloids accurately.

\subsection{Data analysis and statistics}

Curated datasets were handled in the RStudio $R$ software environment using bespoke code and the ggplot2, ggpubr, multcomp, RColorBrewer, Reshape2, rstatix, and tidyverse packages. Quantification of morphology of mouse gastruloids and human gastruloids was based on contour circularity, overall area and inverse aspect ratio. The circularity measurement relates the area and perimeter of the contour to the ratio expected for a circle. The inverse aspect ratio measurement describes the proportions of the gastruloid contour by first bounding its shape within a rectangle. In all cases, the shorter side was divided by the longer side to give a number less than or equal to 1 . This metric therefore measures the similarity to a regular shape: circular contours have a value of 1 while more elongated structures have increasingly small values. Gastruloid length measurements were derived from the longest dimension of the bounding rectangle and are shown for each condition in Supplementary Figs. S6-S8.

The distribution of gastruloid shapes was described by plotting the circularity and inverse aspect ratio measurements of each contour, then thresholding the resulting distribution to classify these data into discrete morphological classes. The thresholds are as follows: spheroid, circularity $>1.5$ (mouse) and $>1$ (human) OR inverse aspect ratio $>1$ (mouse) and $>0.95$ (human); ovoid, circularity 1.25-1.5 (mouse) and 0.94-1 (human) OR inverse aspect ratio 0.95-1 (mouse) and 0.90-0.95 (human); elongated - short, circularity $0.85-1.25$ (mouse) and $0.85-0.94$ (human) OR inverse aspect ratio $0.75-0.95$ (mouse) and $0.75-0.90$ (human); elongated - long, circularity $<0.85$ (mouse, human) OR inverse aspect ratio $<0.75$ (mouse, human). The resulting shape distributions are shown in Supplementary Figs. S9-11.

Statistical comparisons were made between data from the control treatment and each experimental condition for each morphological metric. Statistical testing was conducted with One-Way ANOVA, with Dunnett and Tukey post-hoc testing where appropriate. The results of the Tukey post-hoc tests are shown; a summary that includes the results of the Dunnett tests is available in the supplementary material. A comparable pairwise analysis with Student's $t$-test and Bonferroni correction 

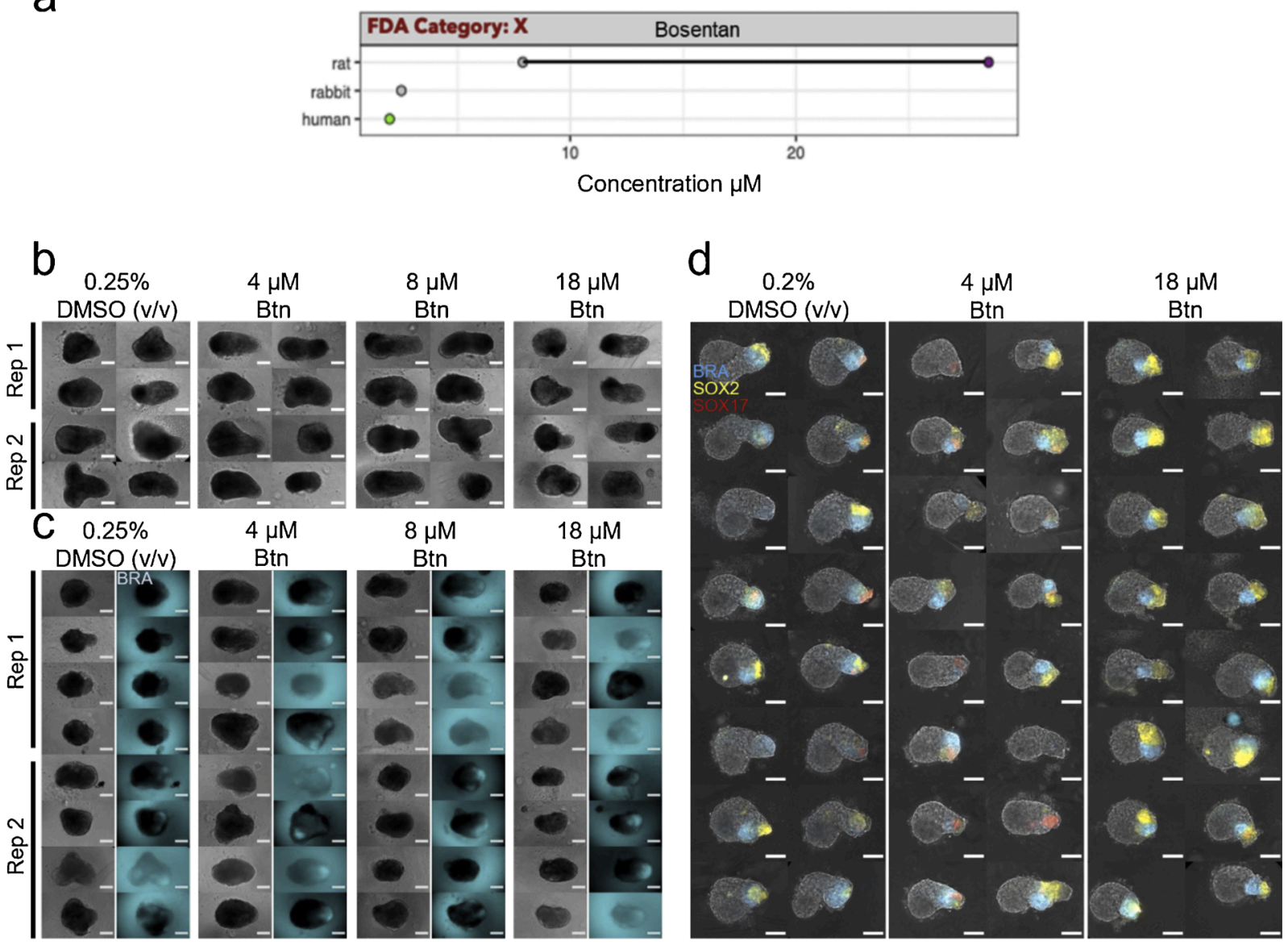

Fig. 6. Bosentan exposure. (a) Literature-based exposure limits in different species in [ $\mu \mathrm{M}]$ (o/o no effect/ NOAEL; o/o teratogenic/ LOAEL, see Suppl. Fig. S1). (bc) E14Tg2A (b) and T/Bra::GFP (c) mouse gastruloids at $120 \mathrm{~h}$, following exposure to DMSO (vehicle control) or bosentan (Btn). Color indicates fluorescent expression of T/Bra::GFP (blue). Representative images from 2 replicates shown. (d) RUES2-GLR human gastruloids at 72 h, following exposure to DMSO or bosentan. Color indicates fluorescent expression of BRA-mCerulean (blue), SOX2-mCitrine (yellow) and SOX17-tdTomato (red). Representative images from 1 replicate shown. Scale bars represent $200 \mu \mathrm{m}$ (b,c) and $100 \mu \mathrm{m}$ (d). Rep, Replicate. (For interpretation of the references to colour in this figure legend, the reader is referred to the web version of this article).

was in agreement with the results of the ANOVA tests. Adjusted p values are indicated with asterisks corresponding to $0<\mathrm{p} \leq 1 \times 10^{\wedge}-4(* * * *), 1$ $\times 10^{\wedge}-4<\mathrm{p} \leq 0.001{ }^{(* * *)}, 0.001<\mathrm{p} \leq 0.01(* *), 0.01<\mathrm{p} \leq 0.05(*)$ and $\mathrm{p}>0.05$ (ns).

The code used for the statistical testing is available online at https: //github.com/peterbeej/Gastruloid-Stats.

\section{Results}

\subsection{All-trans-retinoic acid}

Mouse gastruloids were treated with $0.15 \%$ DMSO (vehicle control) as well as concentrations of $0.4 \mathrm{nM}, 33 \mathrm{nM}$ and $100 \mathrm{nM}$ all-trans retinoic acid (ATRA) (Tables 1, 2). Retinoic acid produced statistically significant differences in gastruloid aspect ratio, circularity and area in both mouse cell lines tested (One-Way ANOVA, $a=0.05$ ). Treatment with 0.4 $\mathrm{nM}$ was sufficient to show significant inhibition of axial extension in mouse gastruloids compared to the controls at $120 \mathrm{~h}$, whereas higher dosages (33 nM, $100 \mathrm{nM}$ ) were associated with drastically reduced size, and gastruloids assumed a spherical shape without any axial elongation (Fig. 2b, c, Fig. 3a, Suppl. Fig. S3a). These observations were consistent across both lines tested, with an apparent loss of T/Bra::GFP expression in all ATRA-treated gastruloids (Fig. 2c). Dosages in excess of $0.4 \mathrm{nM}$ are therefore likely to be cytotoxic or cytostatic in the mouse system.

In contrast to these observations, human gastruloids treated with 0.4 $\mathrm{nM}$ retinoic acid appeared to maintain an equivalent representation of the three germ layers and underwent partial axial elongation (Fig. 2d), although they were still significantly smaller and more circular than controls at $72 \mathrm{~h}$ (Fig. 3b). Concentrations of $33 \mathrm{nM}$ retinoic acid completely inhibited axial extension and promoted the over-expression of SOX2, as observed by fluorescent signal (Fig. 2d). There was no detectable $B R A$ expression across the three replicates. We also observed that, on average, $42 \%$ of human gastruloids treated with $33 \mathrm{nM}$ retinoic acid expressed SOX17 at low levels, compared to $88 \%$ of DMSO controls, where expression was higher. Taken together, these results suggest a lowest observable adverse effect level (LOAEL) of $0.4 \mathrm{nM}$ for both mouse and human gastruloids, albeit with slightly different observable effects.

In comparison, maximum plasma concentrations of the lowest observed adverse effect level (LOAEL Cmax) in rats and rabbits range around $1 \mu \mathrm{M}$ and human therapeutic plasma concentration (human Cmax) is about $1.3 \mu \mathrm{M}$ (Fig. 2a, Table 3) [5]. We therefore compared our 
$a$
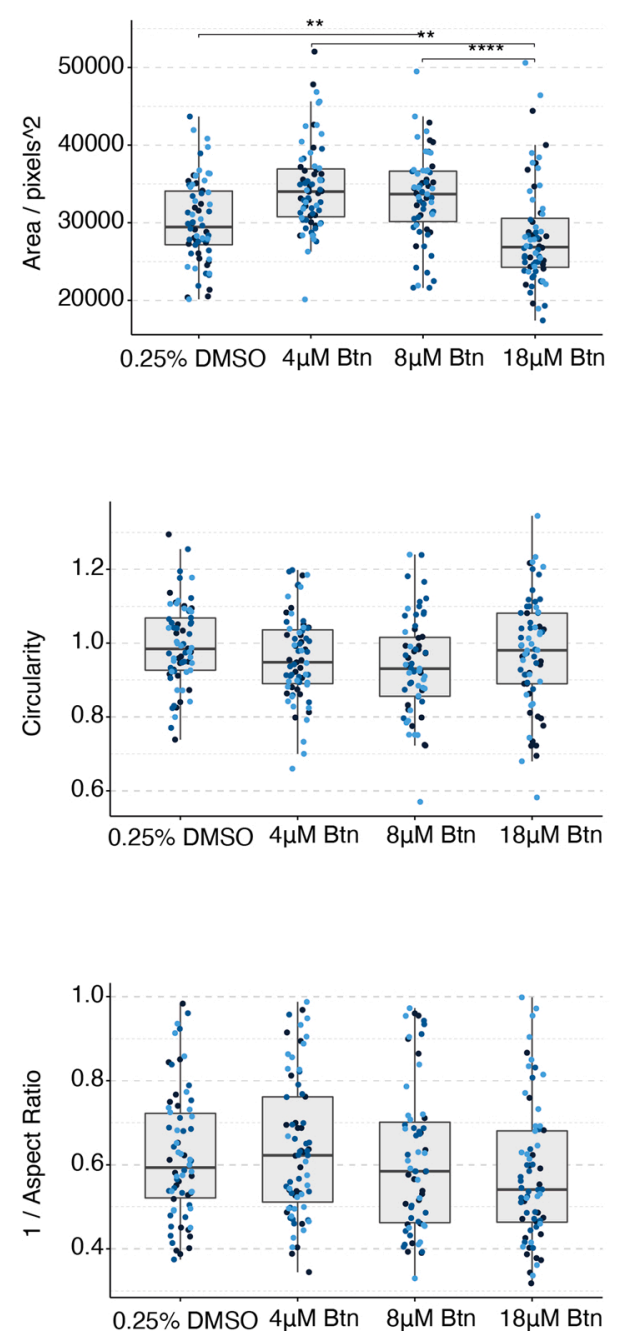
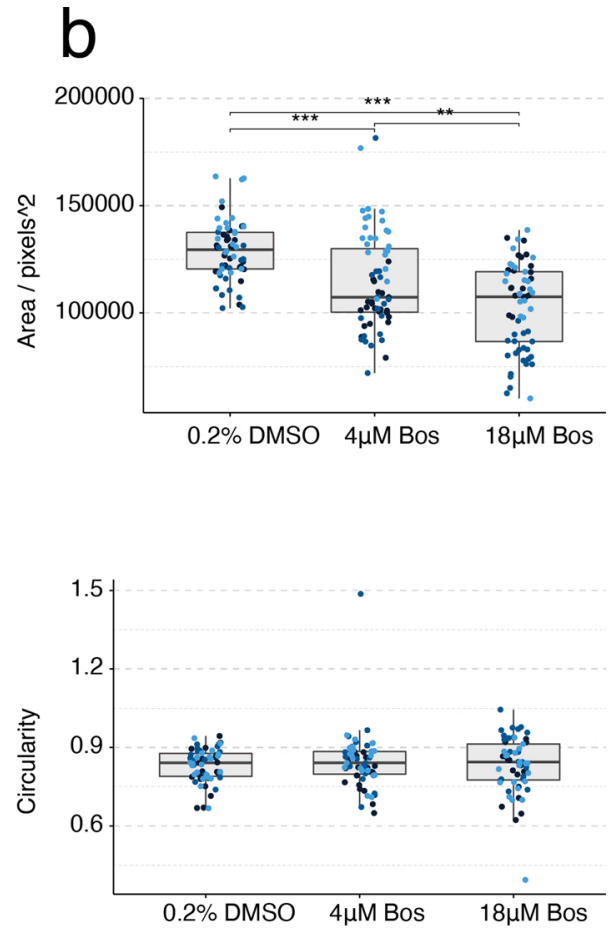

Fig. 7. Gastruloid quantification following bosentan exposure. Quantification of morphology of mouse gastruloids (a), and human gastruloids (b) including Area (top), Circularity (middle) and 1/Aspect Ratio (bottom). Dot colors indicate experimental replicates, and boxplots indicate spread of the data. Significant differences between DMSO (vehicle control) and bosentan (Btn) treatment conditions are indicated in the plots (One-way ANOVA, Tukey's post-hoc test, $a=0.05$ indicated by asterisks (see Materials and Methods for thresholds)). data with in vitro studies of mouse and human embryoid bodies (EB), which revealed $\mathrm{ID}_{50}$ concentrations (half maximal inhibitory concentration of cardiomyocyte differentiation) ranging between $3 \mathrm{nM}$ (mEST) and $\mathrm{TC}_{20}$ concentrations (threshold concentration of $20 \%$ teratogenicity induction based on differential gene expression) of $0.1 \mathrm{nM}$ in hiPSC-derived EBs for ATRA, which corresponds well to the range of the gastruloid assay (Table 3, Suppl. Fig. S2).

\subsection{Valproic acid}

Treatments with $4 \mu \mathrm{M}$ valproic acid seemed to be fairly well tolerated in both the mouse and human gastruloid systems, albeit with a decrease in size in the human (Fig. 5a-b). Valproic acid produced significant changes in gastruloid aspect ratio, circularity and area for the $\mathrm{E} 14 \mathrm{Tg} 2 \mathrm{~A}$ mouse cell line and the RUES2-GLR human cell line (One-Way ANOVA, $a=0.05$ ). T/Bra::GFP mouse gastruloids did not significantly differ in aspect ratio with valproic acid treatment but circularity and area were significantly affected (One-Way ANOVA, $a=0.05$ ). Concentrations higher than $333 \mu \mathrm{M}$ produced clearly spherical mouse gastruloids (Fig. 4b) of reduced size (Fig. 5a), indicating a loss of axial elongation morphogenesis with impaired cell viability or proliferation at $1 \mathrm{mM}$ exposure compared to DMSO controls. These observations were replicated in the T/Bra::GFP cell line, which maintained localised GFP expression after treatment with $4 \mu \mathrm{M}$ valproic acid and did not show significantly increased circularity (Fig. 4c, Suppl. Fig. S3b) at this concentration. Higher concentrations resulted in smaller gastruloids, impaired elongation and a loss of GFP expression.

Human gastruloids treated with $333 \mu \mathrm{M}$ valproic acid also failed to undergo axial elongation morphogenesis. On examining reporter expression, these gastruloids were found to show very low SOX2 expression and elevated BRA expression in comparison to $0.2 \%$ DMSO (v/v) controls (Figs. 4d, 5 b). Valproic acid concentrations of $1 \mathrm{mM}$ seem to induce toxicity in both systems due to strong decrease in gastruloid size. However, this effect would need to be confirmed by cytotoxicity measurements. The overall results indicate a common LOAEL of $333 \mu \mathrm{M}$ for valproic acid (VPA) for both the mouse and human gastruloid systems. Compared to this, VPA studies in the mEST revealed an $\mathrm{ID}_{50}$ of 589 $\mu \mathrm{M}$ for the inhibition of D3 mouse cardiomyocytes, whereas studies in human iPSC derived EBs revealed a $\mathrm{TC}_{20}$ of $85 \mu \mathrm{M}$ (Table 3, Suppl. Fig. S2). By contrast, data for in vivo LOAEL Cmax are $1.6 \mathrm{mM}$ in rat and $6.6 \mathrm{mM}$ in rabbits, whereas human Cmax values are about $1.4 \mathrm{mM}$ (Fig. 4a, Table 3) [5]. 

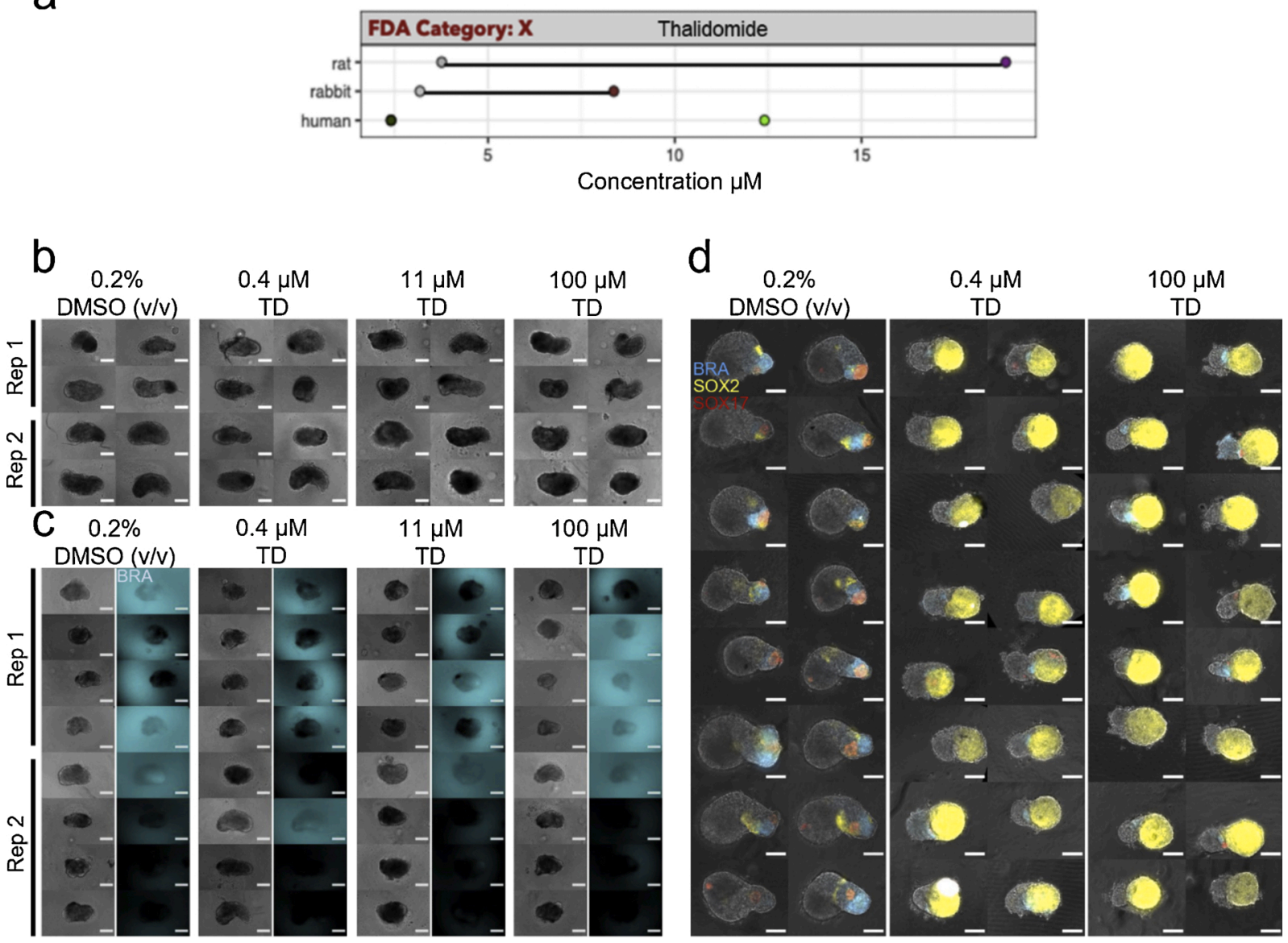

Fig. 8. Thalidomide Exposure. (a) Literature-based exposure limits in different species in $[\mu \mathrm{M}]$ (o/o no effect/ NOAEL; o/o teratogenic/ LOAEL, see Suppl. Fig. S1). (b-c) E14Tg2A (b) and T/Bra::GFP (c) mouse gastruloids at $120 \mathrm{~h}$, following exposure to DMSO (vehicle control) or thalidomide (TD). Color indicates fluorescent expression of T/Bra::GFP (blue). Representative images from 2 replicates shown. (d) RUES2-GLR human gastruloids at $72 \mathrm{~h}$, following exposure to DMSO or thalidomide. Color indicates fluorescent expression of BRA-mCerulean (blue), SOX2-mCitrine (yellow) and SOX17-tdTomato (red). Representative images from 1 replicate shown. Scale bars represent $200 \mu \mathrm{m}$ (b,c) and $100 \mu \mathrm{m}$ (d). Rep, Replicate. (For interpretation of the references to colour in this figure legend, the reader is referred to the web version of this article).

\subsection{Bosentan}

Mouse gastruloids treated with $4-18 \mu \mathrm{M}$ bosentan were slightly larger than controls, but did not appear to be defective in morphogenesis (Fig. 6b, Fig. 7a). Bosentan treatment significantly affected all three metrics (aspect ratio, circularity and area) in the T/Bra::GFP mouse cell line and the RUES2-GLR human cell line (One-Way ANOVA, $a=0.05$ ). The E14Tg2A mouse cell line only showed a significant change in area (One-Way ANOVA, $a=0.05$ ). Gastruloids generated from the $\mathrm{T} / \mathrm{Bra}:$ : GFP cell line cultured in the presence of bosentan showed robust GFP expression that was localised to the pole of the elongations, as observed in untreated controls (Fig. 6c). Morphologically, they were larger and more elongated than controls with $4 \mu \mathrm{M}$ and $8 \mu \mathrm{M}$ treatments (Suppl. Fig. S3c). Bosentan was predicted as a false negative with the mEST. Further investigation of the mouse system will be required to determine whether broader patterns of gene expression are dysregulated under comparable culture conditions. Similarly, human gastruloids treated with $4 \mu \mathrm{M}$ bosentan appeared to maintain representation of all three germ layers and axial elongation morphogenesis, although they were marginally smaller than DMSO-treated gastruloids (Figs. 6d, 7 b).

At $18 \mu \mathrm{M}$, however, the human gastruloids appeared to lose expression of SOX17. We observed that, on average, $33 \%$ showed low levels of SOX17 expression in comparison to $88 \%$ of vehicle controls $(0.2 \%$ DMSO $(\mathrm{v} / \mathrm{v}))$, where expression was higher (Fig. 6d, Suppl. Fig. S4a). The results from the human gastruloid cultures therefore suggest a LOAEL of $18 \mu \mathrm{M}$ bosentan. This corresponds well with the $\mathrm{TC}_{20}$ value for hiPSC-derived embryoid bodies that was determined at $13 \mu \mathrm{M}$. The in vivo LOAEL Cmax for bosentan is about $30 \mu \mathrm{M}$ in rats and it showed no effects in rabbits (Fig. 6a, Table 3) [5].

\subsection{Thalidomide}

Mouse gastruloids treated with $0.4-100 \mu \mathrm{M} \pm$-thalidomide were not significantly altered in either size or morphology (Fig. 8b, c, Fig. 9a, Suppl. Fig. S4b) for both lines tested, with the exception of increased circularity at $100 \mu \mathrm{M}$ concentrations in the E14Tg2A line (One-Way ANOVA, $a=0.05)$. Increasing concentrations appeared to correlate with reduced GFP expression in the T/Bra::GFP cell line $(100 \mu \mathrm{M})$. Thalidomide is false negative predicted with the mEST at concentrations up to 2 $\mathrm{mM}$ and did not show any inhibition of cardiomyocytes. Human gastruloids, on the other hand, showed clearly increased SOX2 expression following exposure to both $0.4 \mu \mathrm{M}$ and $100 \mu \mathrm{M} \pm$-thalidomide (Fig. 8d). 


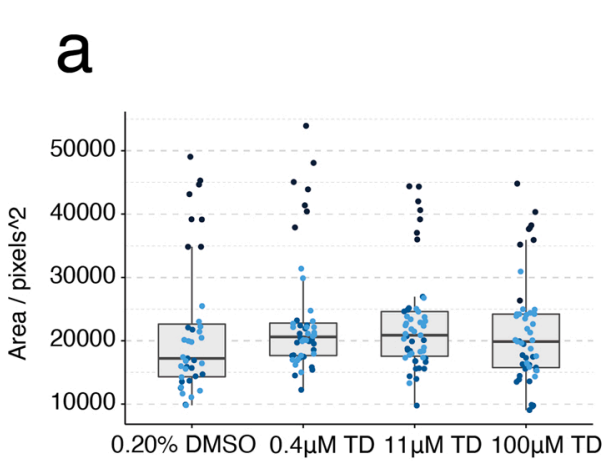

\section{b}

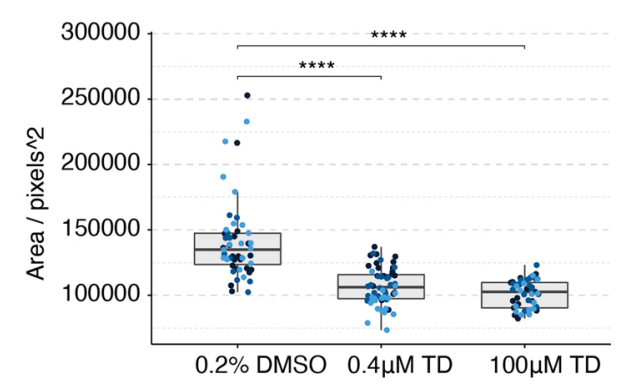

Fig. 9. Gastruloid quantification following thalidomide exposure. Quantification of morphology of mouse gastruloids (a), and human gastruloids (b) including Area (top), Circularity (middle) and 1/Aspect Ratio (bottom). Dot colors indicate experimental replicates, and boxplots indicate spread of the data. Significant differences between DMSO (vehicle control) and thalidomide (TD) treatment conditions are indicated in the plots (One-way ANOVA, Tukey's post-hoc test, $a=0.05$ indicated by asterisks (see Materials and Methods for thresholds)).
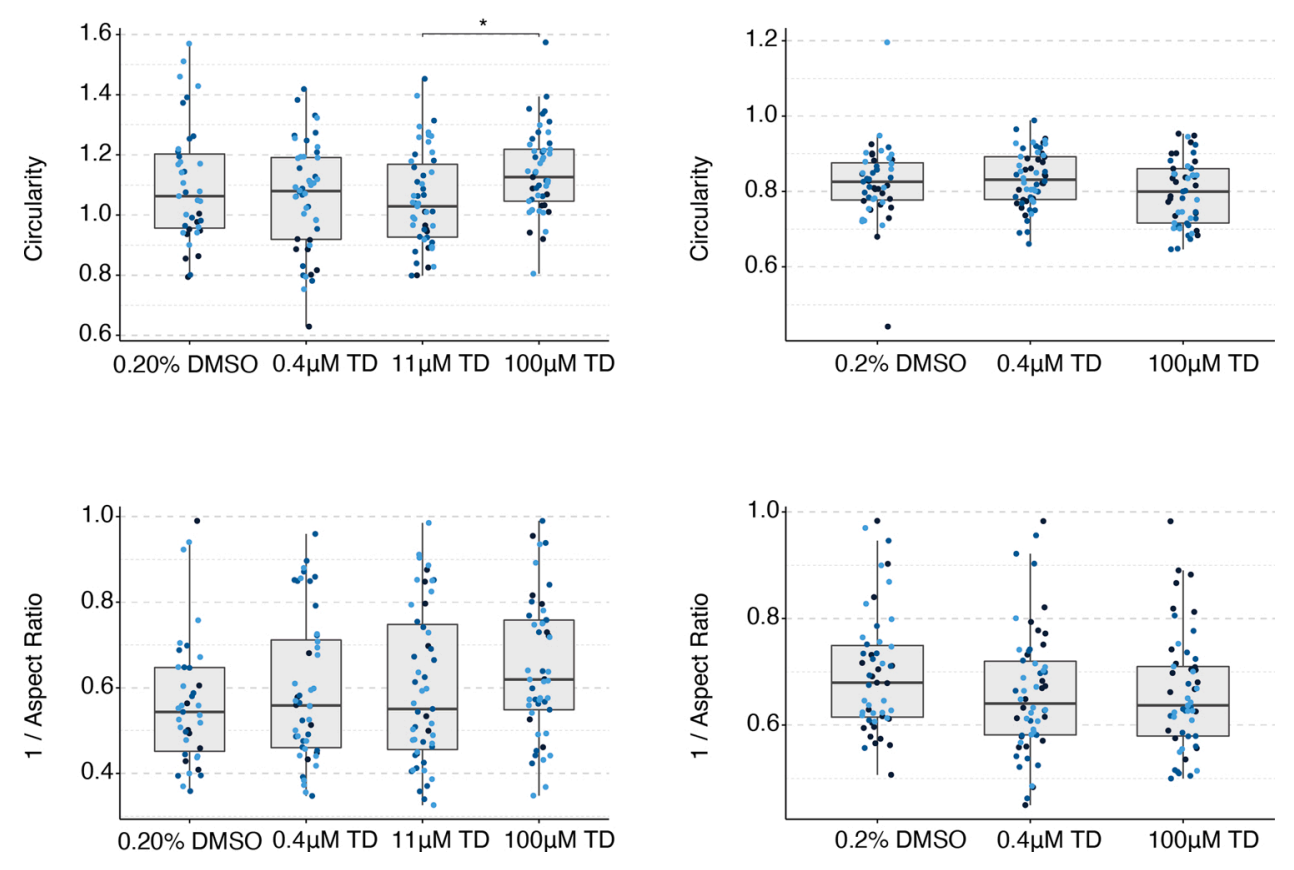

This was accompanied by significant decreases in gastruloid size (Fig. 9b); the One-Way ANOVA analysis of human gastruloid area detected a corresponding change that was significant at the $5 \%$ level. These results suggest a greater sensitivity of the human gastruloid system to exposure to \pm -thalidomide, with a LOAEL for this system defined at $0.4 \mu \mathrm{M}$ in human gastruloids and no observable morphological effect in mouse gastruloids at the concentrations studied. Investigations with the human iPSC derived EB model revealed differential gene expression effects at $\mathrm{TC}_{20}$ concentrations $<0.1 \mu \mathrm{M}$ compared to DMSO vehicle controls (Table 3, Suppl. Fig. S2). In vivo studies revealed a LOAEL Cmax of $19 \mu \mathrm{M}$ in rats and $8.4 \mu \mathrm{M}$ in rabbits [5]. Human therapeutic plasma concentrations are $\sim 2.4 \mu \mathrm{M}$ (Fig. 8a, Table 3). These results therefore suggest that gastruloids could identify species-specific responses to chemical exposure.

\subsection{Phenytoin}

Mouse gastruloids from the E14Tg2a cell line treated with 1-20 $\mu \mathrm{M}$ phenytoin were not significantly different compared to the vehicle-only controls in terms of size and morphology (0.1\% DMSO (v/v); Fig. 10b, c, Fig. 11a, Suppl. Fig. S4c). The $20 \mu \mathrm{M}$ treatment in the T/Bra::GFP line was the only exception, which was significantly smaller and more circular in these data (One-Way ANOVA with Tukey's post-hoc test; $a=$ 0.05). The expression of T/Bra::GFP appeared to be negatively correlated with increasing concentrations of phenytoin ( $>5 \mu \mathrm{M}$, Fig. 10c). Human gastruloids treated with $1-20 \mu \mathrm{M}$ phenytoin maintained proportions and patterns of gene expression that were comparable to controls (Figs. 10d, 11 b), with a significantly reduced area at a concentration of $20 \mu \mathrm{M}$ (One-Way ANOVA, $a=0.05$ ). It would be reasonable to conclude that the $1-20 \mu \mathrm{M}$ concentrations tested fall within the NOAEL (no observable adverse effect level) for these systems. Any follow-up study would be well-advised to use this compound in a different solvent that could allow higher concentrations of phenytoin to be used experimentally without precipitation. Particularly, inhibition of cardiomyocyte-differentiation has already been detected with the mEST at $\mathrm{ID}_{50}$ concentrations of $49 \mu \mathrm{M}$, while with the hiPSC-derived EB model we observed differential gene expression effects at $\mathrm{TC}_{20}$ concentrations of $<8 \mu \mathrm{M}$ for phenytoin. In vivo studies revealed a LOAEL Cmax of about $106 \mu \mathrm{M}$ in rats and $135 \mu \mathrm{M}$ in rabbits [5]. Human therapeutic Cmax is about $57 \mu \mathrm{M}$ (Fig. 10a, Table 3). 
a
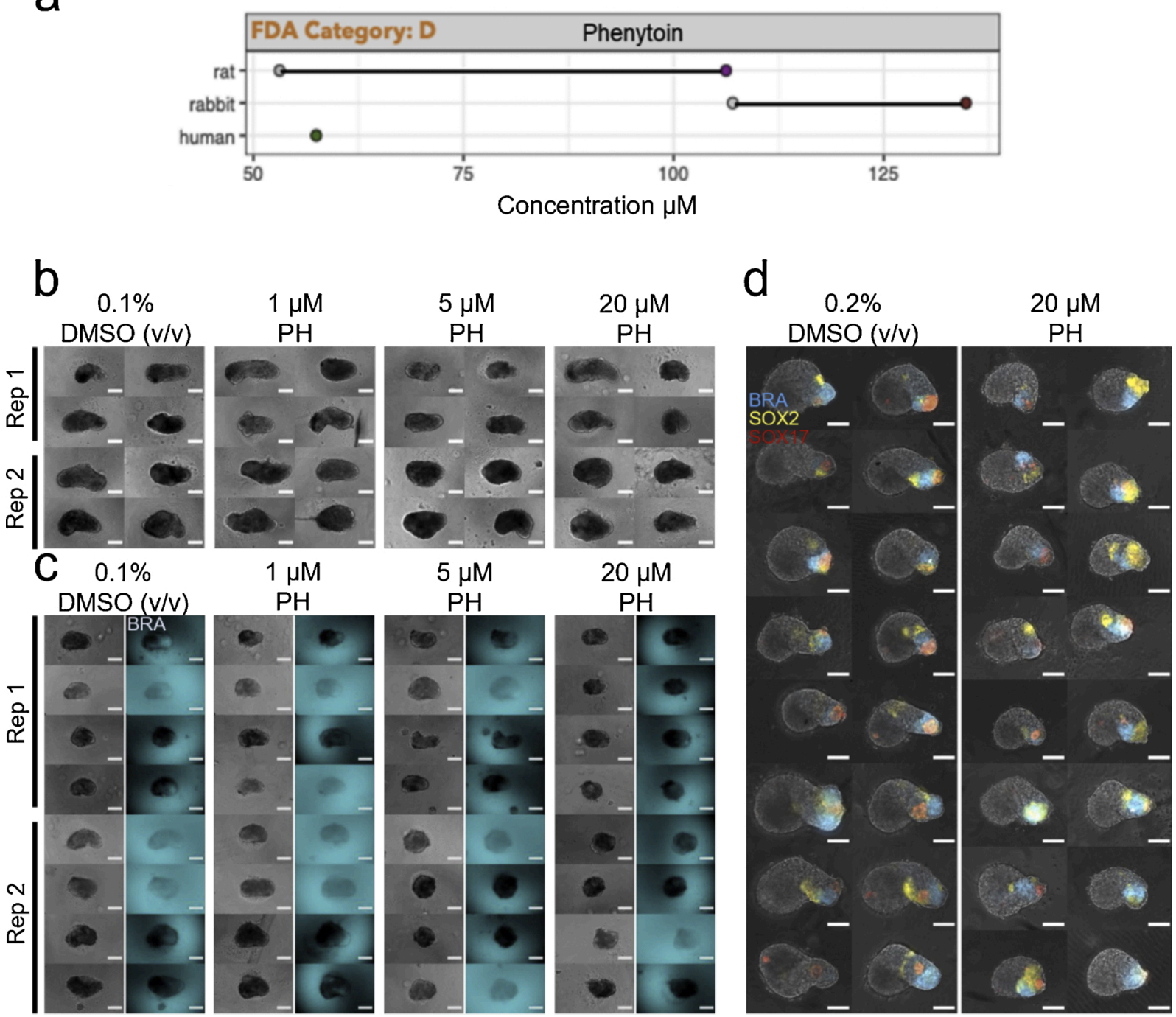

Fig. 10. Phenytoin Exposure. (a) Literature-based exposure limits in different species in $[\mu \mathrm{M}]$ (o/o no effect/ NOAEL; o/o teratogenic/ LOAEL, see Suppl. Fig. S1). (b-c) E14Tg2A (b) and T/Bra::GFP (c) mouse gastruloids at $120 \mathrm{~h}$, following exposure to DMSO (vehicle control) or phenytoin (PH). Color indicates fluorescent expression of T/Bra::GFP (blue). Representative images from 2 replicates shown. (d) RUES2-GLR human gastruloids at $72 \mathrm{~h}$, following exposure to DMSO or phenytoin. Color indicates fluorescent expression of BRA-mCerulean (blue), SOX2-mCitrine (yellow) and SOX17-tdTomato (red). Representative images from 1 replicate shown. Scale bars represent $200 \mu \mathrm{m}(\mathrm{b}, \mathrm{c})$ and $100 \mu \mathrm{m}$ (d). Rep, Replicate. (For interpretation of the references to colour in this figure legend, the reader is referred to the web version of this article).

\subsection{Ibuprofen}

Mouse gastruloids treated with $63-250 \mu \mathrm{M}$ ibuprofen showed no consistent effect on growth or morphology in comparison to vehicleonly controls (0.12\% DMSO (v/v); Fig. 12b, c, Fig. 13a, Suppl. Fig. S5a). While the One-Way ANOVAs identified significant changes in the shape metrics (at the $a=0.05$ level), the significant differences were between drug concentrations that individually were not significantly different from the control treatments. The T/Bra::GFP reporter, however, showed a marked increase in GFP expression with all ibuprofen treatments tested, indicating dysregulated gene expression in comparison to controls (Fig. 12c). Preliminary experiments showed that treatments in the range of $500 \mu \mathrm{M}-2 \mathrm{mM}$ were cytotoxic to both mouse and human embryonic stem cells (data not shown).

On culturing human gastruloids in the presence of $63 \mu \mathrm{M}$ ibuprofen, the expression of SOX2 was marginally elevated compared to vehicleonly controls (0.2\% DMSO (v/v); Fig. 12d). Exposure at both $63 \mu \mathrm{M}$ and $250 \mu \mathrm{M}$ produced gastruloids of reduced size and increased circularity (Fig. 13b). The One-Way ANOVA tests detected significant changes in all three metrics that post-hoc testing identified as significantly different to controls (Tukey's post-hoc test, $a=0.05$ ). It would therefore seem that ibuprofen has a potential effect on expression of $T /$ Bra in mouse and SOX2 in human gastruloids, with a LOAEL at $63 \mu \mathrm{M}$ under these conditions. The mEST predicted ibuprofen as negative whereas the hiPSC-derived EB model determined ibuprofen as positive substance at much higher concentrations, with a $\mathrm{TC}_{20}$ of $855 \mu \mathrm{M}$ (Table 3, Suppl. Fig. S2). The LOAEL Cmax for ibuprofen in rats is about $1.6 \mathrm{mM}$ and no effects have been observed in rabbits [5]. Human therapeutic Cmax is about $286 \mu \mathrm{M}$ (Fig. 12a, Table 3). 
a
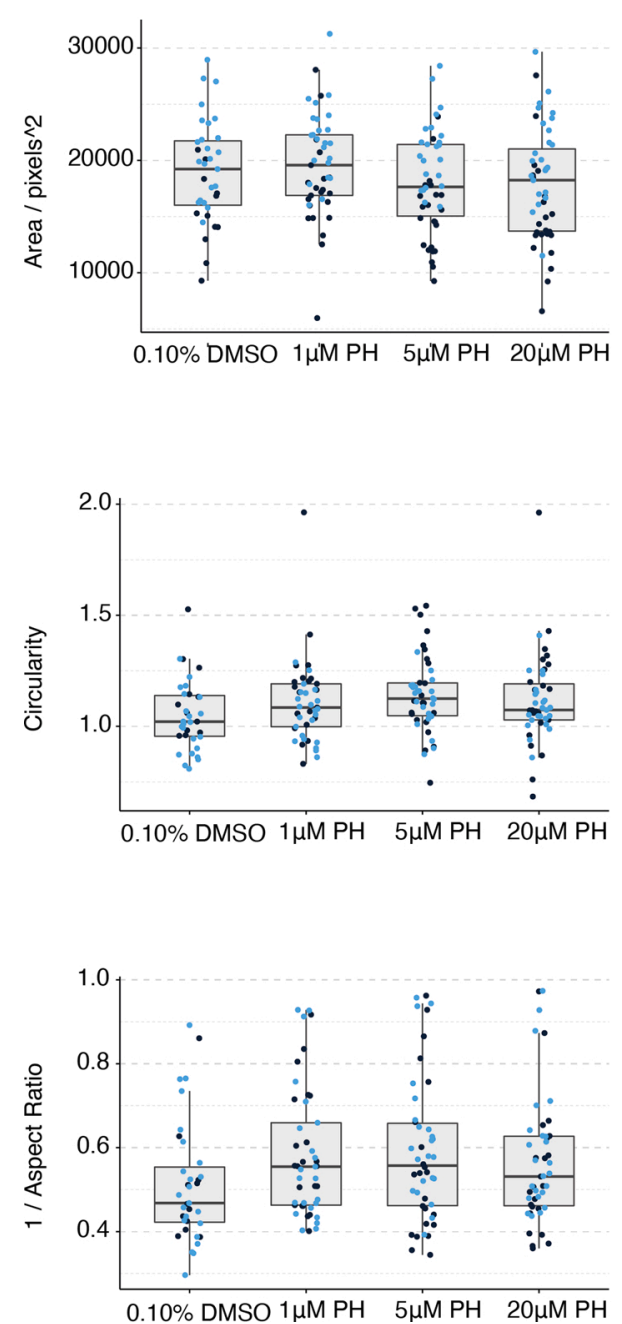

b
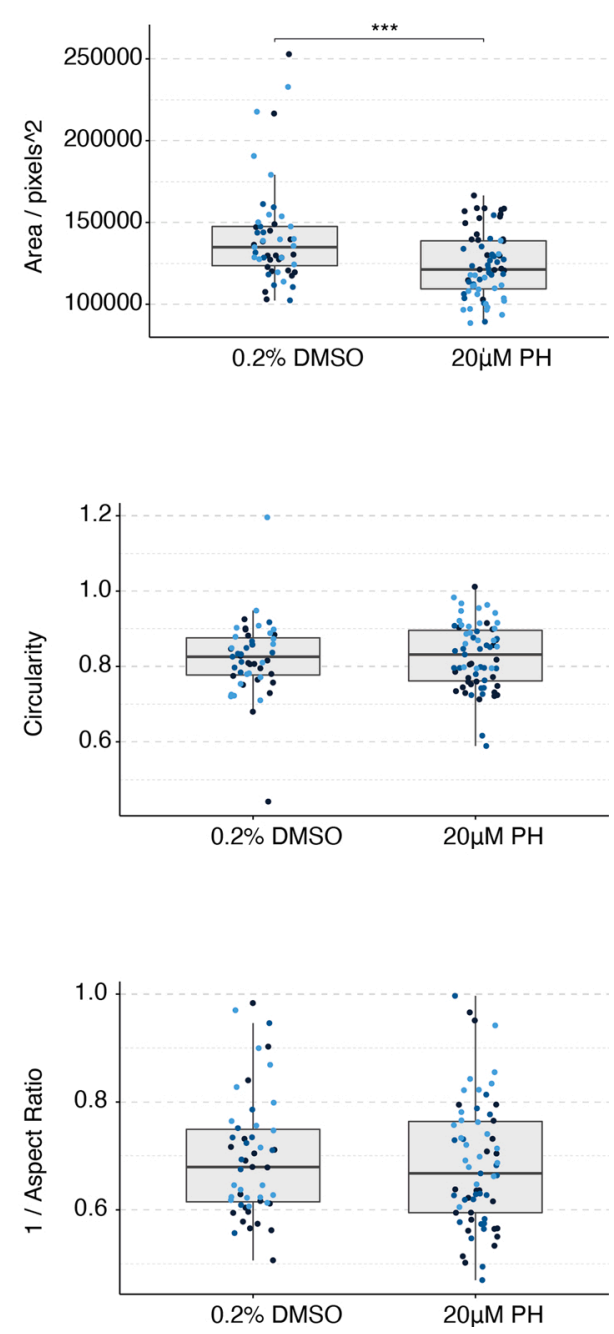

Fig. 11. Gastruloid quantification following phenytoin exposure. Quantification of morphology of mouse gastruloids (a), and human gastruloids (b) including Area (top), Circularity (middle) and 1/Aspect Ratio (bottom). Dot colors indicate experimental replicates, and boxplots indicate spread of the data. Significant differences between DMSO (vehicle control) and phenytoin ( $\mathrm{PH})$ treatment conditions are indicated in the plots (One-way ANOVA, Tukey's post-hoc test, $a=0.05$ indicated by asterisks (see Materials and Methods for thresholds)).

\subsection{Penicillin $G$}

Penicillin was the only putative negative reference compound tested to identify non-specific effects. Penicillin was tested at concentrations of $63 \mu \mathrm{M}, 1 \mathrm{mM}$ and $2 \mathrm{mM}$ (diluted in water) in the mouse and the human gastruloid model (Table 1). Penicillin treatments of E14Tg2A mouse gastruloids induced small morphological changes resulting in slightly larger, more elongated or less circular gastruloids compared to the control (One-Way ANOVA, $a=0.05$ ). In human gastruloids there were minimal effects of increased size at concentrations of $63 \mu \mathrm{M}$ and $1 \mathrm{mM}$ but no other morphological effects were detected (One-Way ANOVA, a $=0.05$ ). No observable adverse effects on reporter gene expression were detected up to the highest concentration levels in both systems (Fig. 14b-c, Fig. 15a-b, Suppl. Fig. S5b). These results are consistent with other in vitro and in vivo systems as well as with human data, where penicillin is not teratogenic within therapeutic concentration levels (Fig. 14a) [5].

\subsection{Gastruloid patterning disruption}

In summary, retinoic acid and valproic acid appear to be similarly teratogenic in both the mouse and the human gastruloid systems. The human system also identifies \pm -thalidomide and bosentan as potentially teratogenic on the basis of altered patterns of reporter gene expression. It would seem that ibuprofen has a more subtle effect in the $63-250 \mu \mathrm{M}$ range, with both systems pointing towards a possible adverse effect at higher concentrations and altered patterns of gene expression across this range. Finally, our results suggest that the range of phenytoin concentrations that were tested falls below the limit of detectable effect in the human system, inviting its reinclusion in any follow-up study (Table 2). The T/Bra::GFP reporter line in the mouse suggests that even these low concentrations of phenytoin may disrupt gene expression.

In general, the observed effects are consistent with the results of our previous study on hiPSC-derived EBs [18]. When examining a comparable range of concentrations, we detected the inhibition of cardiomyocyte differentiation with the MEST and a human hiPSC-derived assay measuring quantitative changes in early developmental gene expression (Table 3, Suppl. Fig. S2). The small panel of reference compounds examined in this study showed variable concentration-dependent effects on gastruloids, with some interesting species-specific differences. Importantly, these differences could be quantified using our image analysis pipeline to identify statistically 

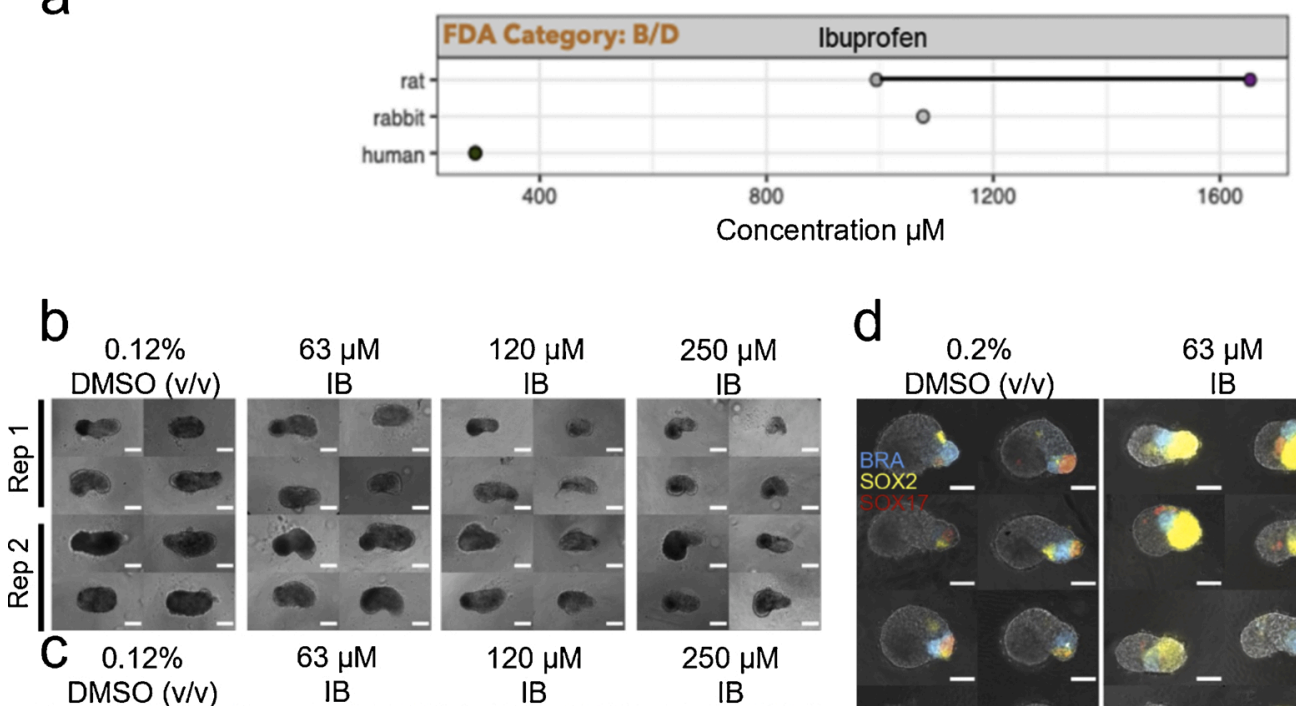

$250 \mu \mathrm{M}$

IB
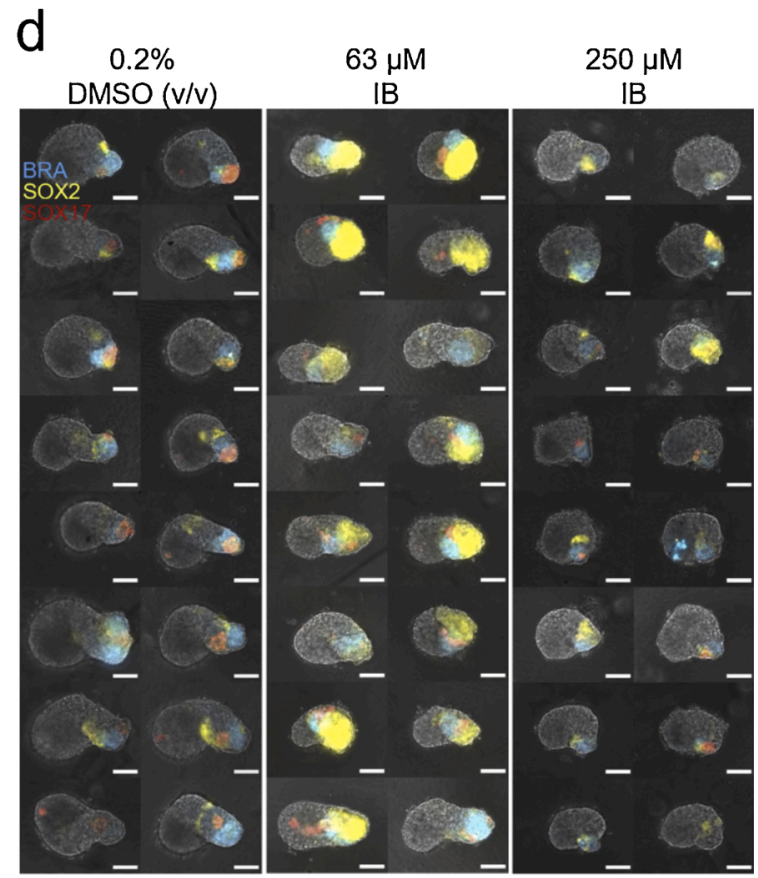

Fig. 12. Ibuprofen Exposure. (a) Literature-based exposure limits in different species in $[\mu \mathrm{M}]$ (o/o no effect/ NOAEL; o/o teratogenic/ LOAEL, see Suppl. Fig. S1). (b-c) E14Tg2A (b) and T/Bra::GFP (c) mouse gastruloids at $120 \mathrm{~h}$, following exposure to DMSO (vehicle control) or ibuprofen (IB). Color indicates fluorescent expression of T/Bra::GFP (blue). Representative images from 2 replicates shown. (d) RUES2-GLR human gastruloids at 72 h, following exposure to DMSO or ibuprofen. Color indicates fluorescent expression of BRA-mCerulean (blue), SOX2-mCitrine (yellow) and SOX17-tdTomato (red). Representative images from 1 replicate shown. Scale bars represent $200 \mu \mathrm{m}$ (b,c) and $100 \mu \mathrm{m}$ (d). Rep, Replicate. (For interpretation of the references to colour in this figure legend, the reader is referred to the web version of this article).

significant changes in morphology. Together these values can be used to develop a simple read-out metric of the assay. Further specification of a defined threshold would enable binary cut-offs to be drawn from the continuous data output that might provide valuable metrics for a generalized assay design (Fig. 16), perhaps using automation to scale-up and randomise treatment conditions.

\section{Discussion}

From this small proof-of-concept study, we conclude that gastruloids might represent a useful tool to assess the effect of compound exposure (in a concentration-dependent manner) on cell differentiation, viability and tissue morphology in a developmental context. This is a particular advantage of using a 3D embryo-like model system with spatiotemporal organization of gene expression, since the gastruloids have intrinsic patterning that mirrors elements of the gastrulating embryo [13,15,24]. Although embryoid body systems reflect cellular differentiation into all three germ layers, the gastruloid systems involve the additional asset of spatiotemporal organisation and morphological rearrangement [18,25, 26]. Here we were able to combine these embryo-like gastruloid models with a toxicological context by assessing morphological perturbations following chemical exposure with multiple phenotypic readouts.

Further experimentation with a wider range of concentrations, a greater number of compounds and different dosing regimens would be required to definitively examine the ability of the gastruloids to provide a comprehensive assay for in vivo prediction of teratogenicity or toxicity. An extension to the work in this manner would allow quantitative estimates of assay specificity, sensitivity and accuracy to be identified, which are currently beyond the scope of a study of this size. Future quantitative metrics could also include analysis of fluorescent reporter intensity, localisation and proportion, that might add an additional layer of detail onto the assay metrics.

However, the results identified here allow for tentative comparisons to be made with existing datasets from animal models or comparative in vitro assays such as EB differentiation. When examined in comparison with such datasets, the gastruloids appear to be able to distinguish between known teratogens (such as valproic acid and retinoic acid) and those that are less teratogenic (ibuprofen) or non-teratogenic (penicillin G) [27-33]. They are even able to recapitulate known species-specific sensitivities such as the human-specific effect of thalidomide [3,4,34] and can highlight subtle changes in developmental gene expression (bosentan, thalidomide, phenytoin). 
a
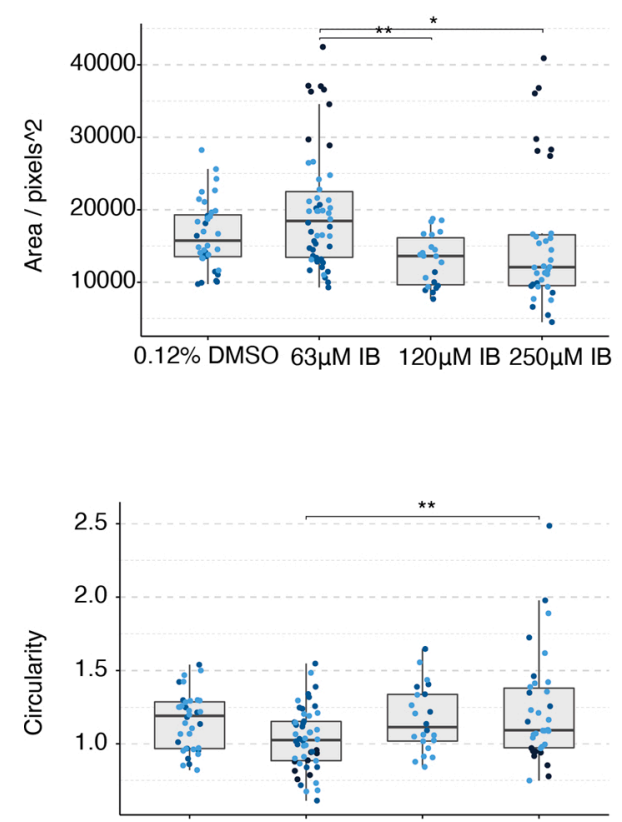

$0.12 \%$ DMSO $63 \mu \mathrm{M}$ IB $120 \mu \mathrm{M}$ IB $250 \mu \mathrm{M}$ IB b
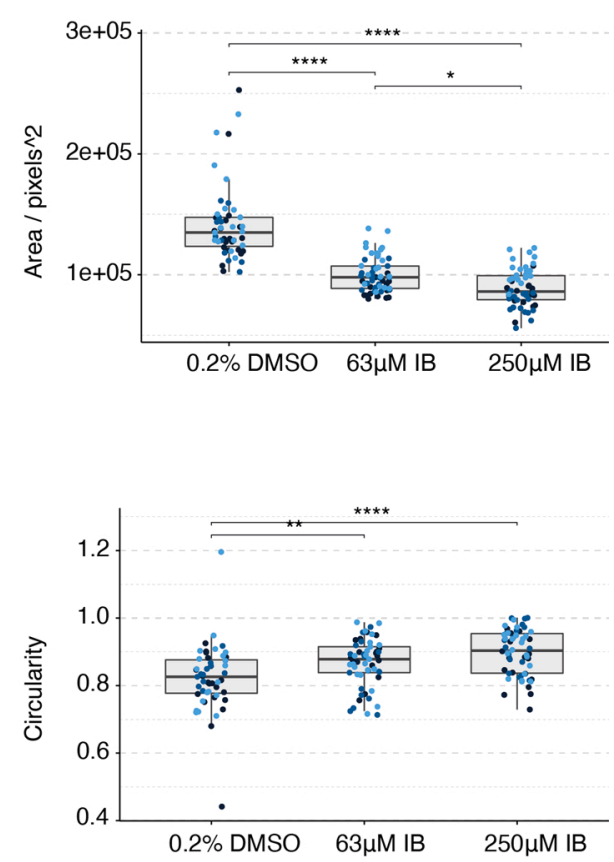

Fig. 13. Gastruloid quantification following ibuprofen exposure. Quantification of morphology of mouse gastruloids (a), and human gastruloids (b) including Area (top), Circularity (middle) and 1/Aspect Ratio (bottom). Dot colors indicate experimental replicates, and boxplots indicate spread of the data. Significant differences between DMSO (vehicle control) and ibuprofen (IB) treatment conditions are indicated in the plots (One-way ANOVA, Tukey's post-hoc test, $a=0.05$ indicated by asterisks (see Materials and Methods for thresholds)).
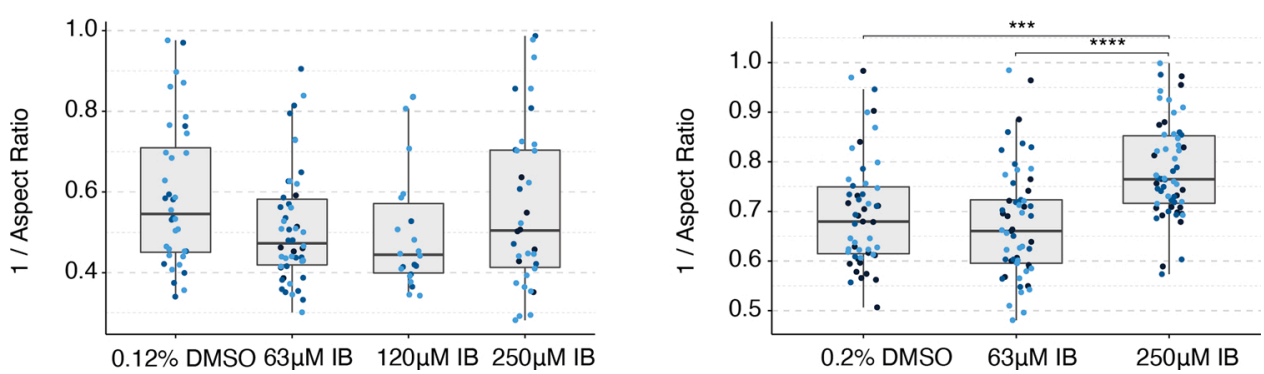

It is clear that there is a range of informative outputs from the assay that describe the effect of the compound, including: morphological shape changes (circularity, lack of elongation, size effects - as seen with lower concentrations of retinoic acid in the mouse system), gene expression effects (such as the decrease in SOX2 expression in human gastruloids following exposure to higher concentrations of valproic acid, which interferes with neurogenesis), and cytotoxicity effects (such as those seen with exposure to high concentrations of retinoic acid or valproic acid in mouse gastruloids) [35]. This suggests that the system can be used to distinguish non-specific effects on cell viability and replication from more specific changes to patterns of gene expression or axial elongation morphogenesis. Together these provide a reference framework of different levels of observable effect that can be used to gauge the response of the system (see Table 2). Additionally the system would benefit from the implementation of a distinct quantitative cytotoxicity measurement to clearly distinguish teratogen from toxic effects.

Interestingly, we also observed that the human gastruloid model has shown to be more sensitive regarding compound exposure in comparison to the mouse gastruloids. This could be due to species-specific effects upon exposure to certain teratogens. Alternatively, the higher sensitivity of the human gastruloids might also be due to a greater informational content of the data by using triple reporters as readout. Different effects might also be caused by different duration of compound exposures to the mouse gastruloids $(120 \mathrm{~h})$ compared to the human gastruloid $(72 \mathrm{~h})$. However, this observation is corroborated by results from previous in vitro studies, performed with mouse and human embryoid body based systems like the mEST and the hiPSC-derived EB model with extended treatment durations, where human EBs tend to be more sensitive to exposures compared to the murine EBs (Table 3).

All teratogens were correctly identified with the human gastruloid model whereas the mouse model only predicted adverse morphological effects for retinoic acid and valproic acid. The mouse model predicted adverse effects on gene expression from \pm -thalidomide, phenytoin and ibuprofen with less pronounced morphological changes. The negative reference compound penicillin G did not cause any adverse effects on reporter gene expression in either model. The lowest observed adverse effect concentration levels where morphological changes were obtained with the gastruloid models were consistently comparable to the inhibitory concentrations of existing mouse $\left(\mathrm{ID}_{50}\right)$ and human in vitro models (TC $_{20}$, Table 3, Suppl. Fig. S2). In this context, we refer to the fact that 


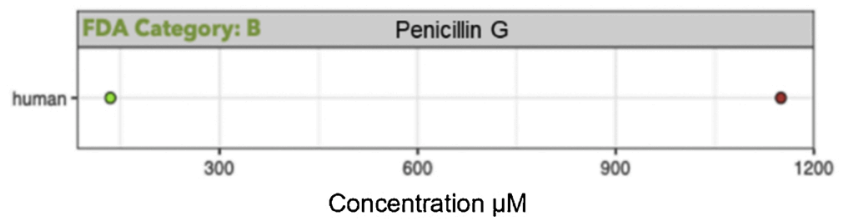

b

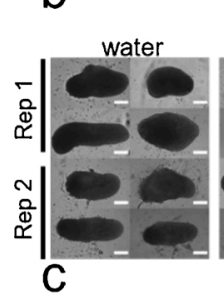

water

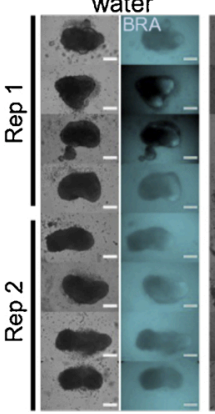

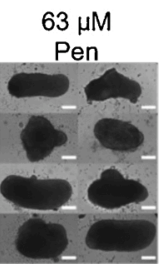

$63 \mu \mathrm{M}$

Pen

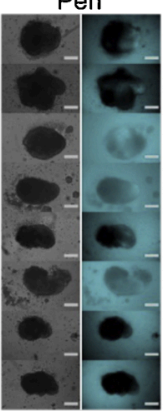

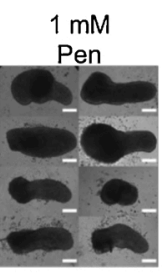

$1 \mathrm{mM}$

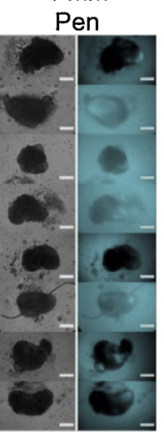

$2 \mathrm{mM}$ Pen

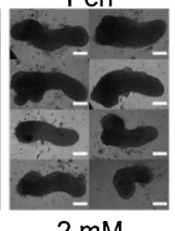

$2 \mathrm{mM}$

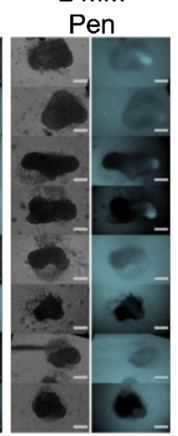

d

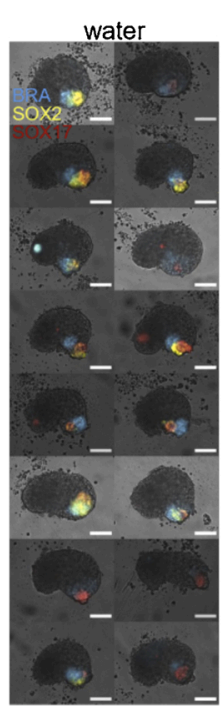

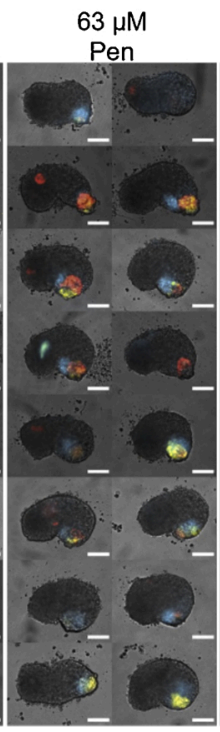

Fig. 14. Penicillin G Exposure. (a) Literature-based exposure limits in different species in $[\mu \mathrm{M}]$ (o no effect/ NOAEL; o teratogenic/ LOAEL, see Suppl. Fig. S1). (bc) E14Tg2A (b) and T/Bra::GFP (c) mouse gastruloids at $120 \mathrm{~h}$, following exposure to water (vehicle control) or penicillin G (Pen). Color indicates fluorescent expression of T/Bra::GFP (blue). Representative images from 2 replicates shown. (d) RUES2-GLR human gastruloids at $72 \mathrm{~h}$, following exposure to water or penicillin G. Color indicates fluorescent expression of BRA-mCerulean (blue), SOX2-mCitrine (yellow) and SOX17-tdTomato (red). Representative images from 1 replicate shown. Scale bars represent $200 \mu \mathrm{m}$ (b,c) and $100 \mu \mathrm{m}$ (d). Rep, Replicate. (For interpretation of the references to colour in this figure legend, the reader is referred to the web version of this article).

both systems were exposed to parental compounds and without any consideration of trans-placental barrier function or maternal metabolism. However, we selected well characterized tool compounds that have been widely described to be either teratogenic or non-teratogenic to human embryos. Moreover, active metabolites can differ in various species and lead to different effects with respect to their toxicological profile. Thus, integration of comprehensive hepatic or barrier functions into the systems might gather more information on the toxicity of active metabolites or compounds that might cross the placenta, which would be in fact highly relevant for investigation on human developmental toxicology.

In this context, it would also be necessary to investigate intracellular compound concentrations or any compound binding to better compare the LOAEL concentrations obtained by both gastruloid models with in vivo parameters and set up translational extrapolation models for pharmacokinetics $[36,37]$. With the limited range of tested concentrations, we observed three to six-fold lower LOAEL concentrations of the gastruloids compared to in vivo Cmax data.

Retinoic acid was an exception, which was detected as positive with more than 2500-fold lower LOAEL concentrations in the mouse gastruloids compared to rat LOAEL. Cmax and human therapeutic plasma concentrations were also about 3000-fold higher than the LOAEL concentration of human gastruloids (Table 3). However, it is necessary to consider that for some compounds, there is a high plasma protein binding (e.g. valproic acid $\geq 90 \%$, phenytoin $90 \%$ ). Thus, free fractions of unbound compound (e.g. VPA $\sim 0.3-0.5 \mathrm{mM}$, phenytoin $\sim 3-9 \mu \mathrm{M}$ ) reflect human therapeutic concentrations more appropriately, when they are set into correlation with free in vitro concentrations of the human gastruloid model, since the E6 human gastruloid medium is free of serum albumin [38-40].

Given the fact that the gastruloids are grown in 96-well plates and assessed using a plate-based imaging system, there is significant scope for automation and increased throughput in gastruloid generation, imaging and analysis if required. Moreover, implementation of an integrated cytotoxicity assessment to predefine concentration ranges that do not cause impaired cell viability but still induce teratogenic effects, would be beneficial for cytotoxic ranges that are not well described. This would subsequently allow for quantitative assessment additional to size determination or circularity of the gastruloids [41,42]. The setup of quantitative thresholds for cytotoxicity and morphological dysregulation will be crucial for the establishment of a comprehensive prediction model.

Towards this end, close examination of the proportion of gastruloids under a threshold value of elongation (arbitrary threshold used for demonstration purposes), gives a quick metric output that could serve as a proxy for morphological effects following compound exposure. Such values could be used to design a simple yes/no pass criteria for a given compound exposure and provide proof-of-concept for the assay (Fig. 16). These thresholds can be determined empirically by contrasting known teratogens to known non-teratogens. Establishing this classification framework will enable the unbiased classification of compounds with unknown teratogenic status and it would be a valuable extension of 
a
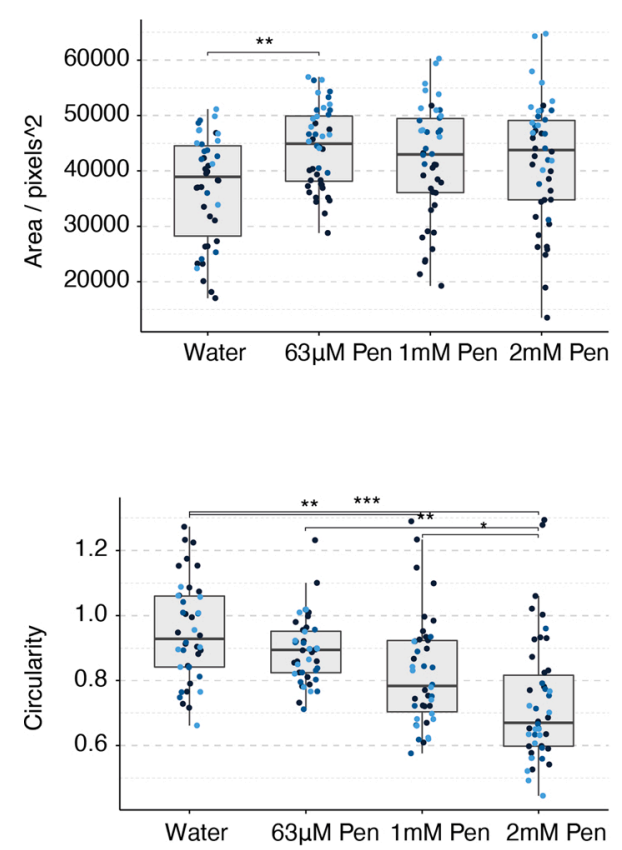

b

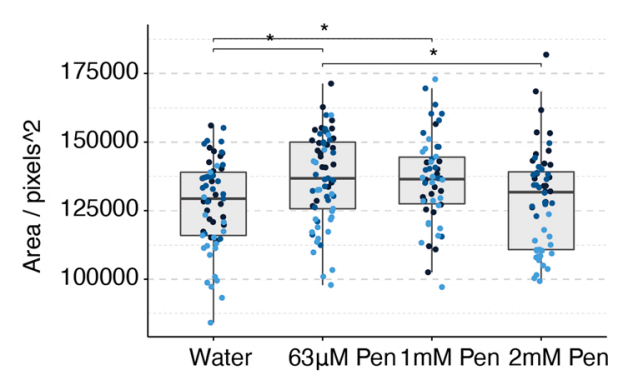

Fig. 15. Gastruloid quantification following penicillin $G$ exposure. Quantification of morphology of mouse gastruloids (a), and human gastruloids (b) including Area (top), Circularity (middle) and 1/Aspect Ratio (bottom). Dot colors indicate experimental replicates, and boxplots indicate spread of the data. Significant differences between water (vehicle control) and penicillin G (Pen) treatment conditions are indicated in the plots (One-way ANOVA, Tukey's post-hoc test, $a=0.05$ indicated by asterisks (see Materials and Methods for thresholds)).

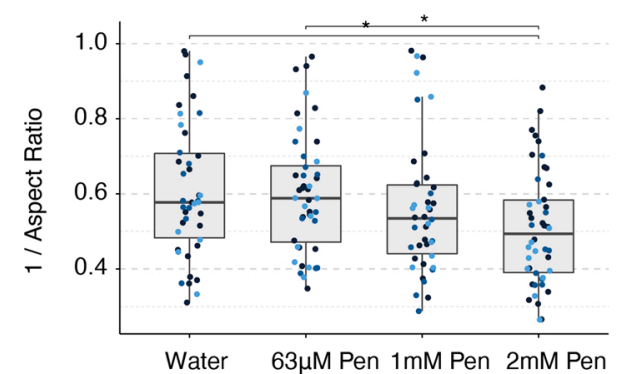

an automated analytical workflow.

\section{Conclusion}

With this study, we have shown that the gastruloid system represents a useful tool for the determination of teratogenic effects during development. Based on a reference panel of seven different compounds we have described morphological and gene expression changes dependent on compound exposure. We were able to differentiate between positive and negative outcomes and even detected species-specific effects. As such, gastruloids represent a novel and potentially powerful assay for teratogenic exposure that utilises the embryo-like spatiotemporal organisation and morphological structure in both mouse and human cell systems. Future efforts could include an extended panel of compounds from different chemical classes with known and unknown teratogenicity, and could establish a clear, quantitative metric for teratogenic and cytotoxic effects as a predictive classifier.

\section{Ethical statement}

The human gastruloid model used in this study does not show any evidence of cell types associated with anterior neural fates, which would be required to form brain tissue, nor do they form extra-embryonic tissues, which would be required for implantation, or show evidence of multi-organ differentiation, which would be necessary for integrated organ system development. Notably, they lack the morphology of an early human embryo, and therefore do not manifest human organismal form. As such, they are non-intact, non-autonomous, and non-equivalent to in vivo human embryos, and do not have human organismal potential. Our research was subject to review and approval from the Human Biology Research Ethics Committee of the University of Cambridge, in compliance with the ISSCR 2016 guidelines.

\section{Declaration of Competing Interest}

Stefan Kustermann reports financial support was provided by $\mathrm{F}$. Hoffmann-La Roche Ltd. Manuela Jaklin reports financial support was provided by F. Hoffmann-La Roche Ltd. Stefan Kustermann reports a relationship with F. Hoffmann-La Roche Ltd that includes: employment. Manuela Jaklin reports a relationship with F. Hoffmann-La Roche Ltd that includes: employment. Alfonso Martinez Arias has patent \#PCT/ GB2019/052670, PCT/GB2019/052668 pending to University of 
a

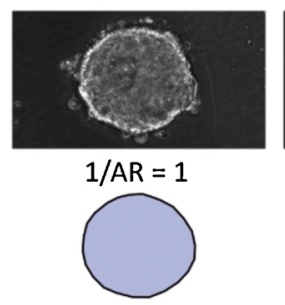

b

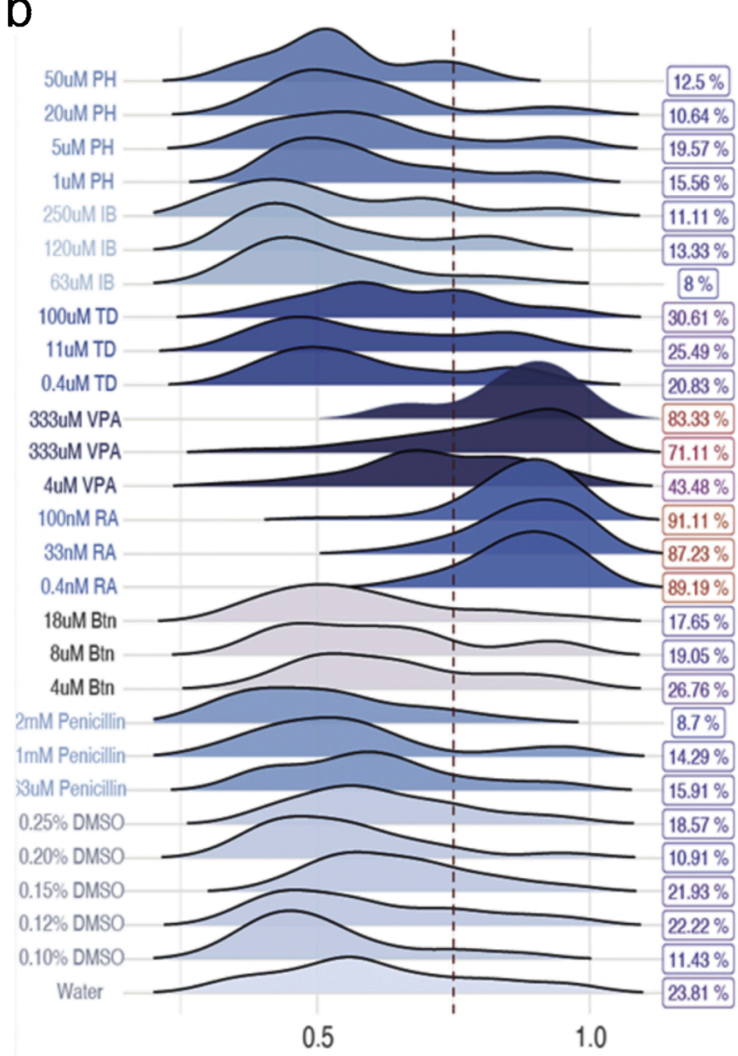

$1 /$ Aspect Ratio

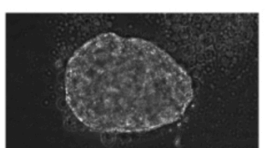

$1 / A R=0.75$

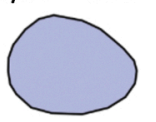

C
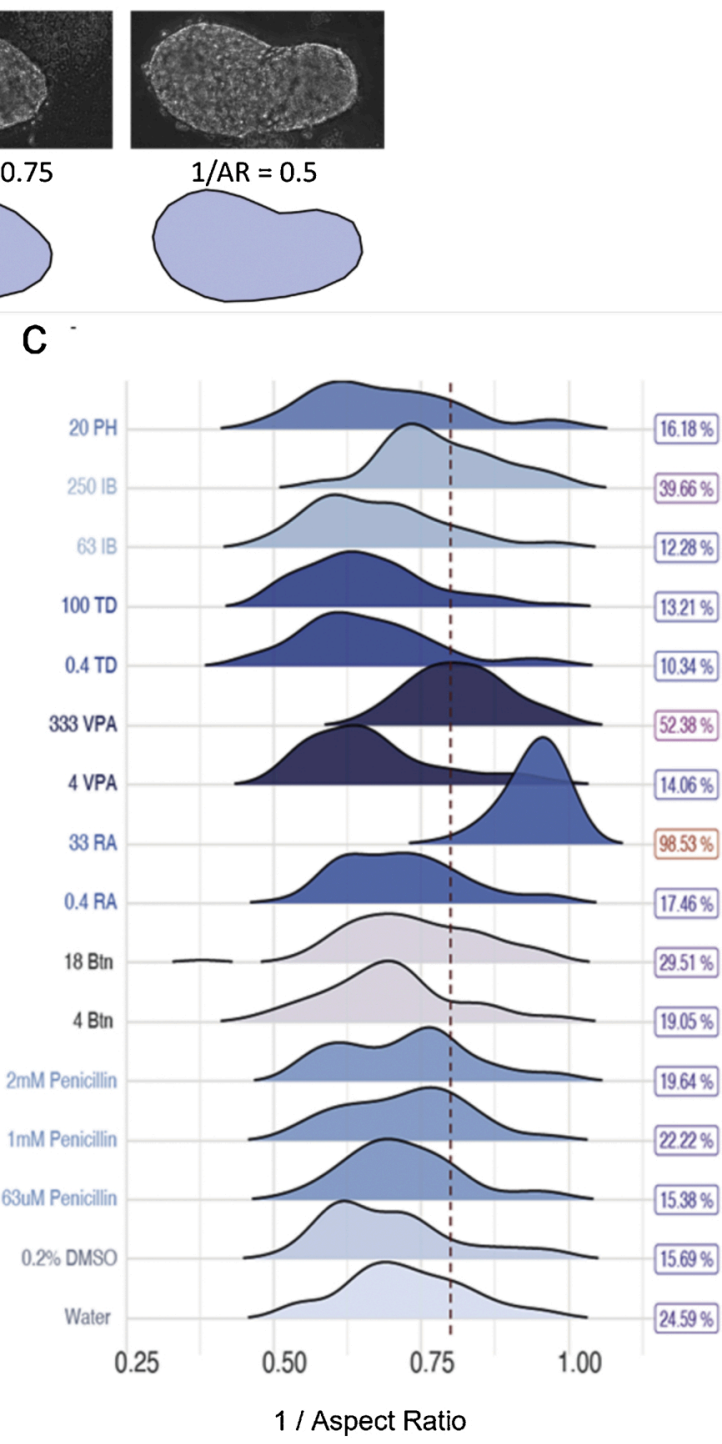

Fig. 16. Using quantitative morphological features as a threshold criteria. (a) Representative images of gastruloids with $1 /$ Aspect Ratio (AR) of $1,0.75$ and 0.5 (top), and their shape outline (bottom). Density plots of mouse gastruloid (b) and human gastruloid (c) length measurements following exposure to each compound. Color indicates treatment compound. Arbitrary 1/Aspect Ratio thresholds of 0.75 for mouse and 0.8 for human gastruloids are drawn (dotted red lines). Percentages (right) of gastruloids above this threshold are shown, and colored by proportion. Btn, Bosentan; RA, Retinoic Acid; VPA, Valproic Acid; TD, Thalidomide; IB, Ibuprofen; PH, Phenytoin. (For interpretation of the references to colour in this figure legend, the reader is referred to the web version of this article).

Cambridge. Naomi Moris has patent \#PCT/GB2019/052670, PCT/ GB2019/052668 pending to University of Cambridge.

\section{Acknowledgments}

The human part of this work was supported by grants from Medical Research Council (MRC) (MR/R017190/1 and MR/V005367/1) to A.M. A. and N.M., a Leverhulme Trust grant (RPG-2018-356) to AMA, as well as a Gates Cambridge Scholarship awarded to V.M.. The mouse part was supported by an ERC Advanced investigator grant (AdG 834580) to A.M. A. supporting P.B.B.. Data derived from human iPSC in vitro model based compound assessment has been supported by the BMBF, EFSA, and the DK-EPA (MST-667-00205) and received funding from the European Union's Horizon 2020 research and innovation program under grant agreements No. 681002 (EU-ToxRisk) and No. 825759 (ENDpoiNTs). The Authors would also like to thank Nicole Schäfer for her valuable contributions in generating all the mEST related data and Paul Barrow for his generous help and useful scientific discussions.

\section{Appendix A. Supplementary data}

Supplementary material related to this article can be found, in the online version, at doi:https://doi.org/10.1016/j.reprotox.2021.08.003.

\section{References}

[1] N. Saunders, S. Liddelow, K. Dziegielewska, Barrier mechanisms in the developing brain, Front. Pharmacol. 3 (46) (2012).

[2] R.C. Gupta, Chapter 1 - introduction, in: R.C. Gupta (Ed.), Reproductive and Developmental Toxicology, second edition, Academic Press, 2017, pp. 3-5.

[3] K.A. Donovan, J. An, R.P. Nowak, J.C. Yuan, E.C. Fink, B.C. Berry, B.L. Ebert, E. S. Fischer, Thalidomide promotes degradation of SALL4, a transcription factor implicated in Duane Radial Ray syndrome, Elife 7 (2018).

[4] M.E. Matyskiela, S. Couto, X. Zheng, G. Lu, J. Hui, K. Stamp, C. Drew, Y. Ren, M. Wang, A. Carpenter, C.W. Lee, T. Clayton, W. Fang, C.C. Lu, M. Riley, P. Abdubek, K. Blease, J. Hartke, G. Kumar, R. Vessey, M. Rolfe, L.G. Hamann, P. P. Chamberlain, SALL4 mediates teratogenicity as a thalidomide-dependent cereblon substrate, Nat. Chem. Biol. 14 (10) (2018) 981-987.

[5] ICH, ICH Harmonized Guideline Detection Of Reproductive And Developmental Toxicity For Human Pharmaceuticals S5(R3), 2020. 
[6] K.C. Brannen, R.E. Chapin, A.C. Jacobs, M.L. Green, Alternative models of developmental and reproductive toxicity in pharmaceutical risk assessment and the 3Rs, ILAR J. 57 (2) (2016) 144-156.

[7] A.E. Seiler, H. Spielmann, The validated embryonic stem cell test to predict embryotoxicity in vitro, Nat. Protoc. 6 (7) (2011) 961-978.

[8] S. Kameoka, J. Babiarz, K. Kolaja, E. Chiao, A high-throughput screen for teratogens using human pluripotent stem cells, Toxicol. Sci. 137 (1) (2014) 76-90.

[9] V. Au-Shinde, S. Au-Klima, P.S. Au-Sureshkumar, K. Au-Meganathan, S. Au-Jagtap, E. Au-Rempel, J. Au-Rahnenführer, J.G. Au-Hengstler, T. Au-Waldmann, J. AuHescheler, M. Au-Leist, A. Au-Sachinidis, Human pluripotent stem cell based developmental toxicity assays for chemical safety screening and systems biology data generation, JoVE (100) (2015), e52333.

[10] J.A. Palmer, A.M. Smith, L.A. Egnash, M.R. Colwell, E.L.R. Donley, F.R. Kirchner, R.E. Burrier, A human induced pluripotent stem cell-based in vitro assay predicts developmental toxicity through a retinoic acid receptor-mediated pathway for a series of related retinoid analogues, Reprod. Toxicol. 73 (2017) 350-361.

[11] A. Warmflash, B. Sorre, F. Etoc, E.D. Siggia, A.H. Brivanlou, A method to recapitulate early embryonic spatial patterning in human embryonic stem cells, Nat. Methods 11 (8) (2014) 847-854.

[12] E.D. Siggia, A. Warmflash, Modeling mammalian gastrulation with embryonic stem cells, Curr. Top. Dev. Biol. 129 (2018) 1-23.

[13] L. Beccari, N. Moris, M. Girgin, D.A. Turner, P. Baillie-Johnson, A.C. Cossy, M. P. Lutolf, D. Duboule, A.M. Arias, Multi-axial self-organization properties of mouse embryonic stem cells into gastruloids, Nature 562 (2018) 272-276.

[14] P. Baillie-Benson, N. Moris, A. Martinez Arias, Pluripotent stem cell models of early mammalian development, Curr. Opin. Cell Biol. 66 (2020) 89-96.

[15] N. Moris, K. Anlas, S.C. van den Brink, A. Alemany, J. Schröder, S. Ghimire, T. Balayo, A. van Oudenaarden, A. Martinez Arias, An in vitro model of early anteroposterior organization during human development, Nature 582 (7812) (2020) 410-415.

[16] S.C. van den Brink, P. Baillie-Johnson, T. Balayo, A.K. Hadjantonakis, S. Nowotschin, D.A. Turner, A. Martinez Arias, Symmetry breaking, germ layer specification and axial organisation in aggregates of mouse embryonic stem cells, Development 141 (22) (2014) 4231-4242.

[17] S. Whitlow, H. Bürgin, N. Clemann, The embryonic stem cell test for the early selection of pharmaceutical compounds, ALTEX-Altern. Anim. Exp. 24 (1) (2007) $3-7$.

[18] M. Jaklin, J.D. Zhang, P. Barrow, M. Ebeling, N. Clemann, M. Leist, S. Kustermann, Focus on germ-layer markers: a human stem cell-based model for in vitro teratogenicity testing, Reprod. Toxicol. 98 (2020) 286-298.

[19] D.A. Turner, Anteroposterior polarity and elongation in the absence of extraembryonic tissues and spatially localised signalling in gastruloids, mammalian embryonic organoids, Development 144 (2017).

[20] M. Hooper, K. Hardy, A. Handyside, S. Hunter, M. Monk, HPRT-deficient (Lesch-Nyhan) mouse embryos derived from germline colonization by cultured cells, Nature 326 (1987).

[21] H.J. Fehling, Tracking mesoderm induction and its specification to the hemangioblast during embryonic stem cell differentiation, Development 130 (2003).

[22] I. Martyn, T.Y. Kanno, A. Ruzo, E.D. Siggia, A.H. Brivanlou, Self-organization of a human organizer by combined Wnt and Nodal signalling, Nature 558 (7708) (2018) 132-135.

[23] N. Moris, K. Anlas, J. Schroeder, S. Ghimire, T. Balayo, S.C. van den Brink, A. Alemany, A. van Oudenaarden, A. Martinez Arias, Generating human gastruloids from Human Embryonic Stem Cells, Protoc. Exch. (2020), https://doi. org/10.21203/rs.3.pex-812/v1.

[24] K.E. Worley, J. Rico-Varela, D. Ho, L.Q. Wan, Teratogen screening with human pluripotent stem cells, Integr. Biol. (Camb.) 10 (9) (2018) 491-501.

[25] A. Mehta, V.B. Konala, A. Khanna, A.S. Majumdar, Assessment of drug induced developmental toxicity using human embryonic stem cells, Cell Biol. Int. 32 (11) (2008) 1412-1424.

[26] M. Simunovic, A.H. Brivanlou, Embryoids, organoids and gastruloids: new approaches to understanding embryogenesis, Development 144 (6) (2017) 976-985.

[27] E.J. Lammer, L.E. Sever, G.P. Oakley Jr, Valproic acid, Teratology 35 (3) (1987) $465-473$.

[28] H. Nau, R.S. Hauck, K. Ehlers, Valproic acid-induced neural tube defects in mouse and human: aspects of chirality, alternative drug development, pharmacokinetics and possible mechanisms, Pharmacol. Toxicol. 69 (5) (1991) 310-321.

[29] E.J. Lammer, D.T. Chen, R.M. Hoar, N.D. Agnish, P.J. Benke, J.T. Braun, C.J. Curry, P.M. Fernhoff, A.W. Grix Jr., I.T. Lott, et al., Retinoic acid embryopathy, N. Engl. J. Med. 313 (14) (1985) 837-841.

[30] H. Nau, Embryotoxicity and teratogenicity of topical retinoic acid, Skin Pharmacol. 6 (Suppl. 1) (1993) 35-44.

[31] S.S. Adams, R.G. Bough, E.E. Cliffe, B. Lessel, R.F.N. Mills, Absorption, distribution and toxicity of ibuprofen, Toxicol. Appl. Pharmacol. 15 (2) (1969) 310-330.

[32] J.S. Dashe, L.C. Gilstrap 3rd, Antibiotic use in pregnancy, Obstet. Gynecol. Clin. North Am. 24 (3) (1997) 617-629.

[33] G.G. Nahum, K. Uhl, D.L. Kennedy, Antibiotic use in pregnancy and lactation: what is and is not known about teratogenic and toxic risks, Obstet. Gynecol. 107 (5) (2006) 1120-1138.

[34] D.G. Belair, G. Lu, L.E. Waller, J.A. Gustin, N.D. Collins, K.L. Kolaja, Thalidomide inhibits human iPSC mesendoderm differentiation by modulating CRBN-dependent degradation of SALL4, Sci. Rep. 10 (1) (2020) 2864.

[35] S. Zhang, W. Cui, Sox2, a key factor in the regulation of pluripotency and neural differentiation, World J. Stem Cells 6 (3) (2014) 305-311.

[36] S.Pa.M. Tettamanti, Testing Times in Toxicology - In Vitro vs In Vivo Testing, ALTEX, 2013.

[37] S.A. Martin, E.D. McLanahan, P.J. Bushnell, E.S. Hunter 3rd, H. El-Masri, Species extrapolation of life-stage physiologically-based pharmacokinetic (PBPK) models to investigate the developmental toxicology of ethanol using in vitro to in vivo (IVIVE) methods, Toxicol. Sci. 143 (2) (2015) 512-535.

[38] M. Gülden, H. Seibert, In vitro-in vivo extrapolation: estimation of human serum concentrations of chemicals equivalent to cytotoxic concentrations in vitro, Toxicology 189 (3) (2003) 211-222.

[39] W. Nasreddine, M. Dirani, S. Atweh, A. Makki, A. Beydoun, Determinants of free serum valproate concentration: a prospective study in patients on divalproex sodium monotherapy, Seizure 59 (2018) 24-27.

[40] J.-M. Hong, Y.-C. Choi, W.-J. Kim, Differences between the measured and calculated free serum phenytoin concentrations in epileptic patients, Yonsei Med. J. 50 (4) (2009) 517-520.

[41] G.P. Daston, B.K. Beyer, E.W. Carney, R.E. Chapin, J.M. Friedman, A.H. Piersma, J. M. Rogers, A.R. Scialli, Exposure-based validation list for developmental toxicity screening assays, Birth Defects Res. B Dev. Reprod. Toxicol. 101 (6) (2014) 423-428.

[42] Y. Marikawa, H.-R. Chen, M. Menor, Y. Deng, V.B. Alarcon, Exposure-based assessment of chemical teratogenicity using morphogenetic aggregates of human embryonic stem cells, Reprod. Toxicol. 91 (2020) 74-91. 